\author{
Universidade de São Paulo \\ Instituto de Física
}

\title{
Fases moduladas em modelos estatísticos com interações quirais
}

\section{William de Castilho}

\author{
Orientador: Prof. Dr. Silvio R. A. Salinas \\ (1) 26 \\ Silvio R. A. Salmás \\ Profector Titular Sépior \\ Ip/stituto de Física \\ Dissertação de mestrado apresentada ao Instituto de Física \\ da Universidade de São Paulo, como requisito parcial para \\ a obtenção do título de Mestre em Ciências.
}

Banca Examinadora:

Prof. Dr. Silvio R. A. Salinas - Orientador (IFUSP)

Prof. Dr. José Ricardo Gonçalves de Mendonça (EACH-USP)

Prof. Dr. Roberto Fernando da Silva Andrade (IF-UFBA)

São Paulo 
FICHA CATALOGRÁFICA

Preparada pelo Serviço de Biblioteca e Informação do Instituto de Física da Universidade de São Paulo

\section{Castilho, William de}

Fases moduladas em modelos estatísticos com interações quirais. São Paulo, 2020.

Dissertação (Mestrado) - Universidade de São Paulo. Instituto de Física. Depto. de Física Geral

Orientador: Prof. Dr. Silvio Roberto de Azevedo Salinas

Área de Concentração: Física Estatística.

Unitermos: 1. Mecânica estatística; 2. Física da matéria condensada; 3 . Mecânica estatística clássica. 


\author{
Universidade de São Paulo
}

Physics Institute

\title{
Modulated phases in statistical models with chiral interactions
}

\author{
William de Castilho
}

Supervisor: Prof. Dr. Silvio R. A. Salinas

Dissertation submitted to the Physics Institute of the University of São Paulo in partial fulfillment of the requirements for the degree of Master of Science.

Examining Committee:

Prof. Dr. Silvio R. A. Salinas - Supervisor (IFUSP)

Prof. Dr. José Ricardo Gonçalves de Mendonça (EACH-USP)

Prof. Dr. Roberto Fernando da Silva Andrade (IF-UFBA) 


\section{Agradecimentos}

Há pessoas demais para agradecer com as poucas linhas que são utilizadas nesse tipo de seção, mas aqui vão mesmos sinceros sentimentos.

Em primeiro lugar, agradecimentos especiais ao Professor Salinas pela orientação e toda ajuda, aprendi muito mais do que esperava com ele. A minha família e amigos de Caçapava e São Paulo, o apoio imparcial deles sempre me manteve. Destacando minha tia, Cida Castilho, que sempre olhou por mim.

Também devo destacar os amigos, Ariel, Harunari e Chico que se dispuseram a me ajudar em várias dúvidas do Mathematica e de programação em geral. Ao Pedro Bittar pelas discussões, caronas e viradas de noite.

O presente trabalho foi realizado com apoio da Coordenação de Aperfeiçoamento de Pessoal de Nível Superior - Brasil (CAPES) - Código de Financiamento 001.

Obrigado a CAPES pelo apoio financeiro.

No mais, obrigado a todos que conviveram comigo durante este tempo. Seu apoio me fez perdurar e seu amor me levou mais alto. 
"Pleasure to me is wonder - the unexplored, the unexpected, the thing that is hidden and the changeless thing that lurks behind superficial mutability. To trace the remote in the immediate; the eternal in the ephemeral; the past in the present; the infinite in the finite; these are to me the springs of delight and beauty." 


\section{Resumo}

Estudamos dois modelos de spins na rede, do tipo Heisenberg clássico com uma escolha discreta dos estados de spin, na presença de interações ferromagnéticas em competição com interações de caráter quiral, do tipo Dzyaloshinskii-Moriya. Na primeira parte desse trabalho, consideramos um modelo de quatro estados apenas, que vamos denominar DM4, e que corresponde ao modelo do relógio quiral, bastante explorado na literatura. Analisamos esse modelo DM4 em diversas situações: (i) em uma dimensão, através da técnica da matriz de transferência, obtendo indicações sobre o estado fundamental do modelo análogo mais realista, em três dimensões: (ii) numa árvore de Cayley, obtendo o diagrama de fases global, com a indicação da existência de estruturas espacialmente moduladas; (iii) numa abordagem de campo médio, em que é simples obter as fronteiras da estrutura desordenada. Usando a experiência adquirida com o modelo DM4, percorremos as mesmas etapas para analisar um modelo de seis estados, ao longo das direções dos eixos cartesianos, que vamos chamar DM6, e que é um "modelo mínimo" para representar um hamiltoniano de Heisenberg com a adição de interações do tipo Dzyaloshinskii-Moriya ao longo de um dos eixos cristalinos. Obtivemos resultados detalhados para o diagrama global de fases no limite de coordenação infinita da árvore de Cayley, incluindo evidências numéricas para mostrar a existência de estruturas fractais, conhecidas como "escadas do diabo". Na parte final desse estudo sobre o modelo DM6, apresentamos em linhas gerais um esboço de solução tipo campo médio. Registramos também um trabalho em andamento, que consiste em definir e analisar uma versão esférica do modelo ferromagnético de Heisenberg com a adição de interações monoaxiais do tipo Dzyaloshinskii-Moriya.

Palavras-chave: Mecânica estatística, Física do estado solido, fases moduladas. 


\section{Abstract}

We studied two classical Heisenberg spin models on a lattice, with a discrete choice of spin states, with ferromagnetic interactions in competition with Dzyaloshinskii-Moriya interactions. In the first part of this work, we consider a model of four states only, which we call DM4, and which corresponds to the chiral clock model, which has been widely explored in the literature. We analyzed this DM4 model in several situations: (i) in one dimension, using the transfer matrix technique, obtaining indications about the ground state of the more realistic counterpart in three dimensions: (ii) on a Cayley tree, which leads to a phase diagram with the indication of the existence of spacial modulated structures; (iii) in a mean-field approach, in which it is relatively simple to obtain the boundaries of the disordered structures. Using the experience we have gained with the DM4 model, we have gone through the same steps to analyze a six-state model, with spin states along the Cartesian directions, which we call DM6, and which is a minimal model for a Heisenberg Hamiltonian with the addition of Dzyaloshinskii-Moriya interactions along one of the crystalline axes. We obtained several results for the global phase diagram in the limit of infinite coordination of a Cayley tree, and presented numerical evidence to show the existence of fractal structures known as "devil's staircases". In the final part of this study on the DM6 model, we sketched a mean-field solution of this problem. Also, we sketched an ongoing work, which is based on defining and analyzing a spherical version of the Heisenberg model with the addition of monoaxial interactions of the Dzyaloshinskii-Moriya type.

Keywords: Statistical mechanics, Solid-state physics, modulated phases. 


\section{Sumário}

1 Introdução $\quad 8$

2 Modelo de quatro estados (DM4) 11

2.1 Modelo DM4 em uma dimensão . . . . . . . . . . . . . . . . . . . . . . 12

2.2 Cálculos na árvore de Cayley . . . . . . . . . . . . . . . 15

2.3 Modelo DM4 na árvore de Cayley . . . . . . . . . . . . . . . . . 18

2.3.1 Limite de coordenação infinita . . . . . . . . . . . . . . . . . . . . 19

2.3.2 Ponto fixo paramagnético . . . . . . . . . . . 20

2.3.3 Cálculos numéricos na árvore de Cayley . . . . . . . . . . . . 21

2.4 Modelo DM4 - solução de campo médio . . . . . . . . . . . . . . . . . . . 22

3 Modelo de seis estados (DM6) 26

3.1 Modelo DM6 em uma dimensão . . . . . . . . . . . . . . . . . . . . . 27

3.1.1 Estruturas de fases no estado fundamental . . . . . . . . . . . 30

3.2 Modelo DM6 na árvore de Cayley . . . . . . . . . . . . . . . . . . . . 31

3.2 .1 Ponto fixo trivial $\ldots \ldots \ldots 33$

3.2.2 Regiões ordenadas do diagrama de fases . . . . . . . . . . . . . 34

3.2.3 Medida da dimensão de Hausdorff . . . . . . . . . . . . . . . . 35

3.3 Modelo DM6 - solução de campo médio . . . . . . . . . . . . . . . . . . 36

3.4 Versão esférica do modelo de Dzyaloshinskii-Moriya . . . . . . . . . . . . . . 40

4 Conclusões $\quad 46$

$\begin{array}{ll}\text { Referências Bibliográficas } & 48\end{array}$ 


\section{Capítulo 1}

\section{Introdução}

Estruturas moduladas são uma ocorrência bem conhecida na física do estado sólido. A descoberta de fases moduladas em ligas de $\mathrm{Cu}-\mathrm{Au}$ data dos anos 1930. Cristais magnéticos com ordenamentos modulados, como o fosfeto de manganês, MnP, foram muito estudados, inclusive no Laboratório de Baixas Temperaturas do IFUSP. O desenvolvimento da mecânica estatística e o estudo de sistemas de spin na rede possibilitaram a proposta de modelos teóricos para dar conta dessas estruturas moduladas. Um das primeiras tentativas de descrever essas modulações foi a proposta do modelo ANNNI, abreviação de "axial next-nearest-neighbor Ising model", que consiste num modelo de spins de Ising numa rede cúbica simples, com interações ferromagnéticas entre sítios primeiros vizinhos e a adição de interações antiferromagnéticas, competitivas, entre spins situados em sítios segundos vizinhos ao longo de uma direção cristalina. Esse modelo ANNNI foi exaustivamente estudado [1] [2] [3], inclusive por nosso grupo aqui na USP [4], dando origem a um dos diagramas de fases mais ricos da literatura, com pontos multicríticos e sequências de fases espacialmente moduladas (associadas a estruturas fractais conhecidas como "escadas do diabo"[5]).

Em paralelo ao modelo ANNNI, com base em investigações pioneiras de Dzyaloshinskii e de Moriya [6] [7], surgiu na literatura uma proposta de competição entre variáveis de spin de caráter vetorial. De acordo com essa proposta, no modelo clássico de Heisenberg, além das interações escalares típicas do ferrromagnetismo, em determinadas circunstâncias devem ser adicionadas "interações quirais"[8], provenientes de um produto vetorial entre duas variáveis de spin em sítios vizinhos, cujo resulado é projetado ao longo de um eixo. Nesse trabalho vamos nos concentrar em modelos simplificados com a adição dessas interações do tipo DzyaloshinskiiMoriya (DM), que são bem menos estudados na literatura, embora tenham interesse crescente 
em magnetismo, e certamente possam ser generalizados para dar conta do comportamento de sistemas bem mais gerais da física dos materiais[9] [10] [11] . Embora o modelo ANNNI tenha sido muito explorado, ainda há espaço para analisar os modelos do tipo Dzyaloshinskii-Moriya (DM), que constituem o tema dessa dissertação. Inicialmente investigamos um modelo tipo DM de 4 estados apenas, com variáveis de spin restritas a quatro estados no plano, que vamos denominar modelo DM4. Embora esse modelo DM4 seja idêntico ao modelo quiral do relógio de quatro estados, cujo diagrama de fases já foi analisado na literatura [12], essa investigação inicial serviu como introdução e treinamento para a abordagem desses problemas. O nosso trabalho central refere-se a um modelo tipo DM de seis estados, que vamos denominar DM6, em que as variáveis de spin são restritas às seis direções dos eixos cartesianos. Esse modelo DM6 talvez seja o "modelo mínimo", fisicamente aceitável, para dar conta das modulações espaciais em sistemas magnéticos com interações do tipo Dzyaloshinskii-Moriya.

Na ausência de campo externo, com interações quirais de caráter monoaxial, uma forma muito utilizada de hamiltoniano de spin do tipo DM é dada por

$$
\mathcal{H}_{D M}=-J_{1} \sum_{\left(\vec{r}, \vec{r}^{\prime}\right)} \vec{S}_{\vec{r}} \cdot \vec{S}_{\vec{r}^{\prime}}-J_{2} \sum_{\left(\vec{r}, \vec{r}^{\prime}\right)} S_{\vec{r}}^{z} S_{\vec{r}^{\prime}}^{z}-D \sum_{(\vec{r}, \vec{r}+\widehat{z})}\left(\vec{S}_{\vec{r}} \times \vec{S}_{\vec{r}+\widehat{z}}\right) \cdot \widehat{z}
$$

em que $\vec{S} \vec{r}$ é um vetor (ou operador vetorial) no sítio $\vec{r}$ de uma rede cristalina, e a competição ocorre entre os termos envolvendo os produtos escalar e vetorial. As duas primeiras somas, sobre sítios primeiros vizinhos, são equivalentes ao termo de troca usual do ferromagneto de Heisenberg com a inclusão de uma anisotropia no espaço dos spins. A terceira soma é sobre primeiros vizinhos ao longo do eixo $z$. Esse terceiro termo, envolvendo um produto vetorial de spins, corresponde às propostas de Dzyaloshinskii e Moriya, que têm sido fartamente investigadas no domínio do magnetismo.

Na versão clássica desse modelo DM, o termo quiral do hamiltoniano, associado ao produto vetorial, pode ser escrito como uma rotação no espaço dos spins em torno do eixo $z$. Isso vai nos permitir uma conexão com os modelos quirais do relógio, que foram muito estudados há alguns anos [12][13]. No caso clássico, é fácil verificar que

$$
\left(\vec{S}_{i} \times \vec{S}_{j}\right) \cdot \widehat{z} \rightarrow \mathbf{S}_{i} \cdot\left(\mathbf{R} \mathbf{S}_{j}\right)
$$

em que $\mathbf{R}$ é uma matriz de rotação,

$$
\mathbf{R}=\left(\begin{array}{ccc}
\cos \theta & \operatorname{sen} \theta & 0 \\
-\operatorname{sen} \theta & \cos \theta & 0 \\
0 & 0 & 1
\end{array}\right)
$$


$\operatorname{Com} \theta=\pi / 2$, recuperamos a forma usual da interação DM (embora também seja interessante considerar uma rotação com um ângulo qualquer $\theta$, fazendo conexões com a quiralidade nos modelos do relógio).

Nos próximos capítulos dessa dissertação vamos apresentar um relato dos nossos estudos sobre duas versões simplificadas da forma monoaxial do hamiltoniano DM, mantendo interações de natureza quiral, mas discretizando as orientações das variáveis de spin:

A. Modelos de spins planares, em que os vetores de spin estão restritos a quatro direções. Esse é o caso do modelo DM4, cuja análise vai funcionar como uma espécie de introdução para o capítulo seguinte.

B. Modelos de 6 estados (DM6), supondo que as variáveis de spin possam assumir seis estados vetoriais, ao longo das direções dos três eixos cartesianos, que é a nossa proposta de "modelo mínino" para as interações do tipo Dzyaloshinskii-Moriya.

Temos a expectativa de verificar a existência de sequências de fases moduladas no diagrama de fases em termos da temperatura (em unidades de $J_{1}>0$ ) e de um parâmetro associado à relevância da quiralidade, $p=D / J_{1}$. 


\section{Capítulo 2}

\section{Modelo de quatro estados (DM4)}

Na primeira parte desse trabalho, consideramos um sistema com spins planares, apontando ao longo de quatro direções, com um termo de troca isotrópico. O hamiltoniano desse modelo DM4 (Dzyaloshinkii - Moriya de quatro estados) é dado por

$$
\mathcal{H}_{D M 4}=-J \sum_{(i, j)} \vec{S}_{i} \cdot \vec{S}_{j}-D \sum_{(i, j)}\left(\vec{S}_{i} \times \vec{S}_{j}\right) \cdot \hat{z},
$$

em que $\vec{S}_{i}$ é um estado de spin no sítio $i$ de uma rede cúbica de $N$ sítios, a primeira soma é sobre sítios vizinhos mais próximos, e a segunda soma é sobre vizinhos mais próximos ao longo da direção $z$ ( $\hat{z}$ é um versor unitário ao longo do eixo). Nesse trabalho vamos tomar sempre $J>0$, mas vamos permitir que o parâmetro $D$ seja positivo ou negativo. Os quatro estados microscópicos de spin são dados por

$$
\vec{S}=\left(\begin{array}{l}
1 \\
0
\end{array}\right),\left(\begin{array}{l}
0 \\
1
\end{array}\right),\left(\begin{array}{c}
-1 \\
0
\end{array}\right),\left(\begin{array}{c}
0 \\
-1
\end{array}\right) .
$$

Esse hamiltoniano de spins, originário do modelo $X Y$ clássico, corresponde à versão quiral do modelo do relógio de 4 estados, que já foi investigada por alguns autores [12].

Vamos desenvolver nosso trabalho ao longo de três linhas:

A1. Estudo de uma versão unidimensional desse modelo. Nesse caso é possível obter uma solução exata através da técnica da matriz de transferência. Embora o sistema não tenha transições de fase, o comportamento no estado fundamental pode ser bastante instrutivo. Definindo o parâmetro de competição,

$$
p=\frac{D}{J},
$$

é possível mostrar que o estado fundamental é ferromagnético para $-1<p<1$, mas se torna 
modulado para $|p|>1$. Quando $p=1$, há uma degenerescência macroscópica, que dá origem a uma entropia residual.

A2. Em seguida, tirando partido da forma monoaxial das interações quirais, formulamos o análogo desse problema na árvore de Cayley. Embora seja possível analisar os resultados para coordenação finita da árvore, apresentamos apenas cálculos no limite de coordenação infinita, que são suficientes para ilustrar a existência de fases espacialmente moduladas e fazer contato com resultados de campo médio.

A3. Finalmente apresentamos em linhas gerais uma solução simples de campo médio, com base na desigualdade de Bogoliubov, mas sem a preocupação de obter resultados numéricos. Fazemos também alguns comentários sobre as conexões com a solução do problema análogo no limite de coordenação infinita da árvore de Cayley.

\subsection{Modelo DM4 em uma dimensão}

Inicialmente vamos considerar o modelo planar de 4 estados, DM4, em uma dimensão. O hamiltoniano desse sistema numa cadeia de $N$ sítios pode ser escrito na forma

$$
\mathcal{H}_{1 D M 4}=-J \sum_{i=1}^{N}\left[S_{i}^{x} S_{i+1}^{x}+S_{i}^{y} S_{i+1}^{y}\right]-D \sum_{i=1}^{N}\left[S_{i}^{x} S_{i+1}^{y}-S_{i}^{y} S_{i+1}^{x}\right],
$$

em que os estados microscópicos de spin são dados pela equação (2.2). Vamos agora utilizar o formalismo da matriz de transferência, com condições periódicas de contorno. Definindo novas variáveis, um pouco mais compactas, dadas por

$$
K=\exp (\beta J) ; \quad \Delta=\exp (\beta D)
$$

em que $\beta=1 / k_{B} T$ é o inverso da temperatura, escrevemos a matriz de transferência

$$
\mathbf{T}_{4}=\left(\begin{array}{cccc}
K & \Delta & K^{-1} & \Delta^{-1} \\
\Delta^{-1} & K & \Delta & K^{-1} \\
K^{-1} & \Delta^{-1} & K & \Delta \\
\Delta & K^{-1} & \Delta^{-1} & K
\end{array}\right)
$$

Notando que $\mathbf{T}_{4}$ é uma matriz cíclica, de período 4 , há procedimentos bem conhecidos para encontrar os seus autovalores, que podem ser escritos em termos das raízes quárticas da unidade. Vamos escrever essas raízes quárticas na forma

$$
(1)^{1 / 4} \Longrightarrow \rho_{k}=\exp \left(i \frac{2 \pi}{4} k\right)
$$


com $k=0,1,2,3$. Então os quatro autovalores de $\mathbf{T}_{4}$ serão dados por

$$
\lambda_{k}=K\left(\rho_{k}\right)^{0}+\Delta\left(\rho_{k}\right)^{1}+K^{-1}\left(\rho_{k}\right)^{2}+\Delta^{-1}\left(\rho_{k}\right)^{3} .
$$

De forma explícita, podemos escrever

$$
\begin{gathered}
\lambda_{0}=K+\Delta+K^{-1}+\Delta^{-1}=2 \cosh \beta J+2 \cosh \beta D, \\
\lambda_{1}=K+i \Delta-K^{-1}-i \Delta^{-1}=2 \sinh (\beta J)+2 i \sinh (\beta D), \\
\lambda_{2}=K-\Delta+K^{-1}-\Delta^{-1}=2 \cosh \beta J-2 \cosh \beta D
\end{gathered}
$$

e

$$
\lambda_{3}=K-i \Delta-K^{-1}+i \Delta^{-1}=2 \sinh (\beta J)-2 i \sinh (\beta D)
$$

em que fica bem clara a simetria $D \longleftrightarrow-D$ para $J>0$. Examinando essas expressões, é imediato perceber que $\lambda_{0}$ é o maior autovalor para qualquer temperatura finita $(\beta \neq \infty)$. De fato, $\lambda_{0}$ é real e maior do que $\lambda_{2}$; além disso, os autovalores $\lambda_{1}$ e $\lambda_{3}$ são complexos conjugados, mas com módulo sempre menor do que $\lambda_{0}$. Portanto, no limite termodinâmico o autovalor $\lambda_{0}$ define a energia livre desse sistema, que não apresenta nenhum tipo de singularidade.

Embora não haja anomalias termodinâmicas para temperaturas finitas, é interessante examinar o estado fundamental desse sistema. Quando a temperatura $T$ tende a zero $(\beta \rightarrow \infty)$, lembrando as definições de $K$ e $\Delta$, dados pela equação (2.5), e tomando valores positivos do parâmetro $p=D / J$, temos os limites

$$
\begin{aligned}
& \lambda_{0} \rightarrow K+\Delta, \\
& \lambda_{1} \rightarrow K+i \Delta, \\
& \lambda_{2} \rightarrow K-\Delta
\end{aligned}
$$

$\mathrm{e}$

$$
\lambda_{3} \rightarrow K-i \Delta
$$

Potanto, para $0<p<1$, o estado fundamental é quatro vezes degenerado, correpondendo às quatro possibilidades de ordenamento ferromagnético ao longo dos eixos no plano. Para $p>1$, os termos dependentes de $\Delta$ são dominantes, e aparecem autovalores complexos, que indicam ordenamento espacialmente modulado. Para $T \longrightarrow 0$ e $p=1$, os autovalores com módulo 2 indicam a coexistência de muitas fases, com entropia residual proporcional a $\ln 2$. 
A função de partição no limite termodinâmico é dada por

$$
Z_{1 D M 4} \sim \lambda_{0}^{N}
$$

de onde obtemos a energia interna por sítio da rede,

$$
u=\lim _{N \rightarrow \infty}-\frac{1}{N} \frac{\partial \ln \left(Z_{1 D M 4}\right)}{\partial \beta}=-\frac{-D e^{-D \beta}+D e^{D \beta}-J e^{-J \beta}+J e^{\beta J}}{e^{-D \beta}+e^{\beta D}+e^{-J \beta}+e^{J \beta}} .
$$

Utilizando essa expressão, no limite de temperaturas muito baixas, obtemos o gráfico desenhado na figura 1 , em que representamos $u / J$ contra o parâmetro $p=D / J$, e que confirma a nossa análise do estado fundamental.

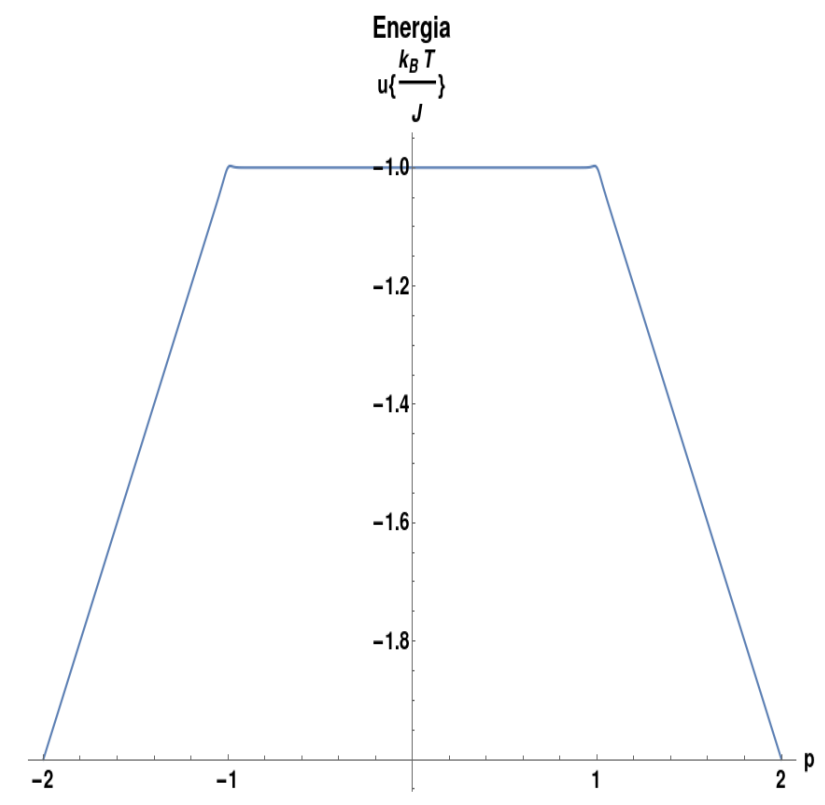

Figura 2.1: Energia interna u (em unidades de $\mathrm{J}$ ) contra $p=D / J$, para temperaturas, $k_{B} T / J$, suficientemente baixas. Note a mudança de comportamento para $p=D / J= \pm 1$.

Também é fácil obter uma expressão para a entropia por sítio, que nós utilizamos para desenhar o gráfico da figura 2, para temperaturas suficientemente baixas. Esse gráfico ilustra a existência do ponto de multifase para $p=1$. 


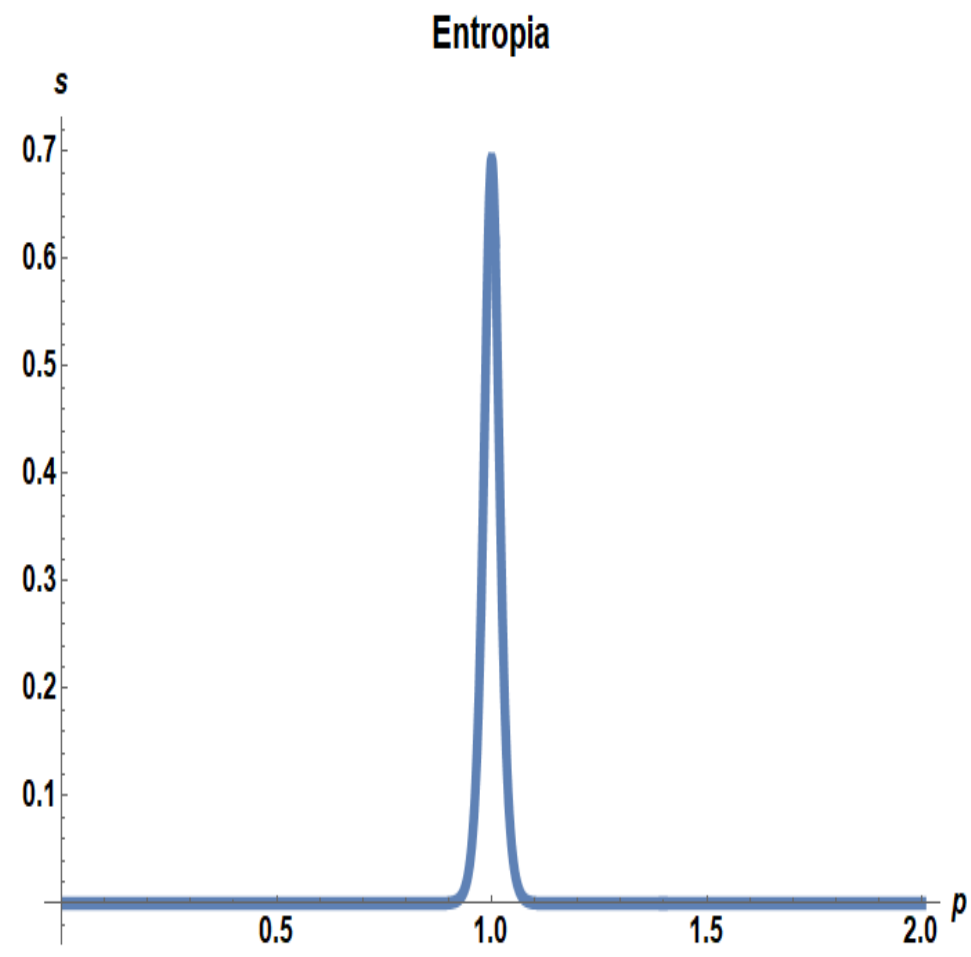

Figura 2.2: Entropia s, em unidades da constante de Boltzmann $k_{B}$, contra o parâmetro $p=D / J$, para temperaturas, $k_{B} T=J$, suficientemente baixas. Note o comportamento nas vizinhanças do ponto de multifase, $\operatorname{com} p=1$.

Fazendo com cuidado o limite de baixas temperaturas, com $J>0$, obtemos

$$
\lim _{T \rightarrow 0} u=-J
$$

para $-1<D / J<1$. Caso contrário, ou seja, com $|D|>J$, obtemos

$$
\lim _{T \rightarrow 0} u=-D
$$

para $D>0$, ou

$$
\lim _{T \rightarrow 0} u=D,
$$

para $D<0$, de acordo com a ilustração da figura 1 .

Para a entropia, obtemos o limite $s(T \rightarrow 0) \rightarrow 0$, para qualquer valor da razão $p=D / J$, exceto em $p=1$, que é um ponto de multifase, em que obtemos uma entropia residual,

$$
s(T \rightarrow 0) \rightarrow k_{B} \ln (2),
$$

que está de acordo com a ilustração da figura 2. 
Para descobrir as fases no estado fundamental devemos construir as estruturas mais simples que dão origem às energias que foram encontradas. Para $|D|<J$, temos quatro possibilidades,

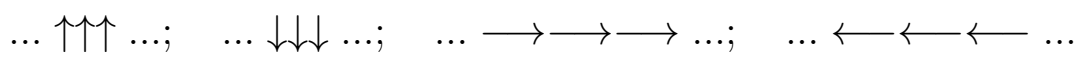

Para $|D|>J$, temos fases espacialmente moduladas,

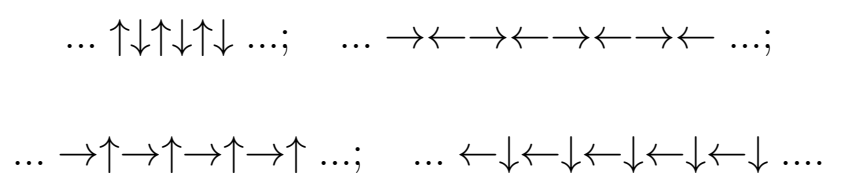

Para $|D|=J$, temos um grande número de possibilidades.

\subsection{Cálculos na árvore de Cayley}

Ao invés de formular o problema estatístico numa rede de Bravais (por exemplo, numa rede cúbica) decidimos explorar as características físicas do modelo definido em uma estrutura mais símples, a árvore de Cayley, que é basicamente um grafo sem ciclos fechados. O modelo de Ising numa árvore de Cayley, de coordenação z, dá origem a um problema de mapeamento não linear. No caso ferromagnético, os atratores desse mapeamento são pontos fixos muito simples, que correspondem a soluções do problema estatístico no interior profundo da rede. Embora a árvore de Cayley seja muito diferente de uma rede de Bravais, os resultados de interesse físico são qualitativamente semelhantes[14].

Vamos considerar algumas gerações de uma rede de Cayley de coordenação $z=3$. 


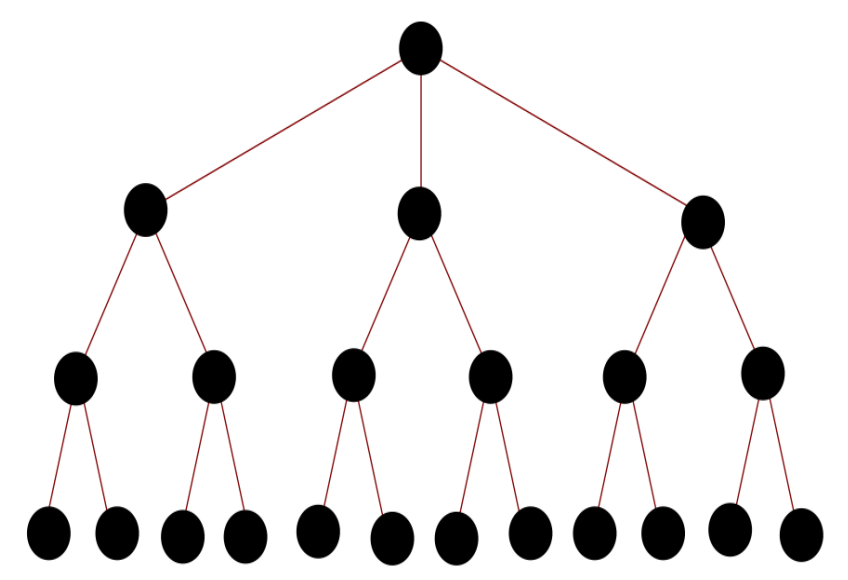

Figura 2.3: Árvore de Cayley de coordenação 3 com três gerações. Imagem gerada com o programa fornecido em: https://demonstrations.wolfram.com/KCayleyTrees/.

Na figura 2.3, indicamos um sítio central (o elemento no topo), que chamamos de geração $\mathrm{N}$, que é sequencialmente ligado a três outros elementos (geração $N-1$ ). Cada elemento da geração $N-1$ está ligado a dois outros elementos da geração $\mathrm{N}-2$, e assim por diante. Não há ciclos fechados nessa estrutura.

Vamos considerar a fomulação do problema simples (no contexto do ensemble canônico) do modelo de Ising ferromagnético na ausência de um campo, numa árvore de Cayley de coordenação z (ramificação $r=z-1$ ). O hamiltoniano de spin é dado por

$$
\mathcal{H}=-J \sum_{\{i, j\}} \sigma_{i} \sigma_{j}
$$

em que $\sigma= \pm 1$ são as variáveis de spin nos sítios da rede, e a soma é sobre pares de sítios primeiros vizinhos. Com essas considerações podemos escrever as relações de recorrência

$$
\begin{aligned}
& Z_{l+1}^{+}=\left\{\exp [\beta J] Z_{l}^{+}+\exp [-\beta J] Z_{l}^{-}\right\}^{r} \\
& Z_{l+1}^{-}=\left\{\exp [-\beta J] Z_{l}^{+}+\exp [\beta J] Z_{l}^{-}\right\}^{r}
\end{aligned}
$$

em que $r=z-1$ é a ramificação da rede, $Z_{l}^{+}$e $Z_{l}^{-}$são funções parciais de partição, que têm um elemento da geração 1 como seu centro; os sinais + e - indicam qual valor de spin é 
assumido pelo elemento central. Com essas funções podemos definir uma magnetização efetiva por partícula,

$$
m_{l}=\frac{+Z_{l}^{+}-Z_{l}^{-}}{Z_{l}^{+}+Z_{l}^{-}}
$$

Definindo também $\Omega=Z_{l}^{+}+Z_{l}^{-}$, temos

$$
\begin{gathered}
\frac{Z_{l}^{+}}{\Omega}=\frac{m_{1}+1}{2}, \\
\frac{Z_{l}^{-}}{\Omega}=\frac{-m_{1}+1}{2} .
\end{gathered}
$$

Substituindo nas relações (2.23) e (2.24), chegamos a

$$
m_{l}=\frac{A^{r}-B^{r}}{A^{r}+B^{r}}
$$

em que

$$
\begin{aligned}
& A=\cosh (\beta J)+m_{l-1} \sinh (\beta J), \\
& B=\cosh (\beta J)-m_{l-1} \sinh (\beta J) .
\end{aligned}
$$

Após algumas manipulações algébricas, na tradição de análise dos sistemas dinâmicos, escrevemos a relação de recorrência para as magnetizações efetivas,

$$
m_{l}=f\left(m_{l-1}\right)=\tanh \left\{r \tanh ^{-1}\left[(\tanh \beta J) m_{l-1}\right]\right\}
$$

em que $l=1,2,3, \ldots$. Os atratores dessa relação correspondem às soluções de interesse físico (no interior profundo da árvore de Cayley). É fácil perceber que sempre há atrator trivial, um ponto fixo com magnetização nula, $m^{*}=0$, que corresponde à solução paramagnética do sistema físico (fase desordenada). No caso do modelo ferromagnético, com $J>0$, também há um ponto fixo $m^{*} \neq 0$, pelo menos para valores suficientemente baixos da temperatura. No caso de um modelo antiferromagnético, com $J<0$, o atrator não trivial é um ciclo-dois da relação de recorrência). Cálculos desse tipo são bem conhecidos na literatura desde, ao menos, 1982 [15].

Vamos agora fazer uma análise da estabilidade dos pontos fixos, dados pela equação

$$
m^{*}=f\left(m^{*}\right)=\tanh \left\{r \tanh ^{-1}\left[(\tanh \beta J) m^{*}\right]\right\} .
$$

Mantendo apenas termos lineares, escrevemos a expansão

$$
m_{l}-m^{*}=\left(\frac{\partial f}{\partial m}\right)_{m=m^{*}}\left(m_{l}-m^{*}\right)+\ldots=\Lambda\left(m_{l}-m^{*}\right)+\mathcal{O}\left[\left(m_{l}-m^{*}\right)^{2}\right],
$$


em que

$$
\Lambda=\frac{r \tanh (\beta J) \operatorname{sech}^{2}\left[r \tanh ^{-1}\left(m^{*} \tanh \beta J\right)\right]}{1-\left(m^{*} \tanh \beta J\right)^{2}} .
$$

A estabilidade do ponto fixo $m^{*}$ é garantida pela condição $|\Lambda|<1$.

O ponto fixo desordenado, $m^{*}=0$, que existe para qualquer temperatura, é estável apenas para

$$
\Lambda=r \tanh (\beta J)<1
$$

ou seja, para temperaturas suficientemente altas,

$$
\frac{k_{B} T}{J}>2\left[\ln \left(\frac{z}{z-2}\right)\right]^{-1} .
$$

O limite de estabilidade desse ponto fixo corresponde exatamente à temperatura crítica prevista pela aproximação de Bethe-Peierls para o ferromagneto de Ising numa rede hipercúbica simples de coordenação $z$. No limite de coordenação infinita, $z \rightarrow \infty, J \rightarrow 0$, com $z J$ fixo, nós recuperamos a temperatura crítica conhecida da aproximação de campo médio.

Essa análise é um pouco mais envolvida para $m^{*} \neq 0$. Supondo que $m^{*}$ seja muito pequeno, podemos escrever a expansão

$$
m^{*}+\frac{1}{3}\left(m^{*}\right)^{3}+\ldots=r \tanh (\beta J) m^{*}+\frac{r}{3} \tanh ^{3}(\beta J)\left(m^{*}\right)^{3}+\ldots,
$$

de onde obtemos o termo dominante,

$$
\left(m^{*}\right)^{2}=\frac{3 r^{2}}{r^{2}-1}[r \tanh (\beta J)-1]
$$

que somente existe para

$$
r \tanh (\beta J)>1,
$$

ou seja, na região de temperaturas em que a solução desordenada é instável. Não é difícil mostrar que essa solução ferromagnética é estável em toda essa região, ou seja, para

$$
\frac{k_{B} T}{J}<2\left[\ln \left(\frac{z}{z-2}\right)\right]^{-1} .
$$

A coincidência dos limites de estabilidade dos dois tipos de ponto fixo, paramagnético e ferromagnético, indica que a transição é contínua (ou de segunda ordem).

\subsection{Modelo DM4 na árvore de Cayley}

De acordo com os procedimentos explicados na subseção anterior, formulamos o problema estatístico do modelo DM4 numa árvore de Cayley de ramificação $r \geq 1$. Considerando um 
elemento da rede, que pode assumir cada um dos quatro estados de spin, obtivemos as relações de recorrência

$$
\begin{aligned}
& Z_{1}^{\prime}=\left(e^{\beta J} Z_{1}+e^{\beta D} Z_{2}+e^{-\beta J} Z_{3}+e^{-\beta D} Z_{4}\right)^{r}, \\
& Z_{2}^{\prime}=\left(e^{-\beta D} Z_{1}+e^{\beta J} Z_{2}+e^{\beta D} Z_{3}+e^{-\beta J} Z_{4}\right)^{r}, \\
& Z_{3}^{\prime}=\left(e^{-\beta J} Z_{1}+e^{-\beta D} Z_{2}+e^{\beta J} Z_{3}+e^{\beta D} Z_{4}\right)^{r}, \\
& Z_{4}^{\prime}=\left(e^{\beta D} Z_{1}+e^{-\beta J} Z_{2}+e^{-\beta D} Z_{3}+e^{\beta J} Z_{4}\right)^{r},
\end{aligned}
$$

em que $Z_{i}^{\prime}$, para $i=1, \ldots, 4$ são as funções de partição parciais associadas às quatro possibilidade do nó inicial, e $Z_{i}$, para $i=1, \ldots, 4$, são as funções correspondentes dos primeiros vizinhos na geração imediatamente seguinte da árvore de Cayley. Os atratores desse mapeamento (que nesse caso não se restringem a pontos fixos) correspondem às soluções de interesse físico do problema, no interior profundo da árvore de Cayley.

Agora é conveniente definir uma magnetização por partícula,

$$
\vec{m}=\langle\vec{S}\rangle=\left(\begin{array}{l}
m_{x} \\
m_{y}
\end{array}\right)=\frac{\vec{S}(1) Z_{1}+\vec{S}(2) Z_{2}+\vec{S}(3) Z_{3}+\vec{S}(4) Z_{4}}{Z_{1}+Z_{2}+Z_{3}+Z_{4}},
$$

e uma grandeza $\vec{q}$, correspondente a uma média das componentes do vetor de spin ao quadrado,

$$
\vec{q}=\left(\begin{array}{c}
\left\langle S_{x}^{2}\right\rangle \\
\left\langle S_{y}^{2}\right\rangle
\end{array}\right)=\left(\begin{array}{c}
q_{x} \\
q_{y}
\end{array}\right)
$$

Portanto, temos

$$
\begin{aligned}
& m_{x}=\frac{Z_{1}-Z_{3}}{\Omega}, \\
& m_{y}=\frac{Z_{2}-Z_{4}}{\Omega}, \\
& q_{x}=\frac{Z_{1}+Z_{3}}{\Omega}, \\
& q_{y}=\frac{Z_{2}+Z_{4}}{\Omega},
\end{aligned}
$$

em que $\Omega=Z_{1}+Z_{2}+Z_{3}+Z_{4}$. Essas definições implicam que $q_{y}=1-q_{x}$. Com algumas manipulações algébricas chegamos às expressões

$$
\frac{Z_{1}}{\Omega}=\frac{q_{x}+m_{x}}{2}, \frac{Z_{2}}{\Omega}=\frac{q_{y}+m_{y}}{2}, \frac{Z_{3}}{\Omega}=\frac{q_{x}-m_{x}}{2}, \frac{Z_{4}}{\Omega}=\frac{q_{y}-m_{y}}{2} .
$$

Portanto, o mapeamento fica reduzido a três equações de recorrência apenas,

$$
m_{x}^{\prime}=\frac{A_{1}^{r}-A_{3}^{r}}{A_{1}^{r}+A_{2}^{r}+A_{3}^{r}+A_{4}^{r}}
$$




$$
\begin{aligned}
m_{y}^{\prime} & =\frac{A_{2}^{r}-A_{4}^{r}}{A_{1}^{r}+A_{2}^{r}+A_{3}^{r}+A_{4}^{r}}, \\
q_{x}^{\prime} & =\frac{A_{1}^{r}+A_{3}^{r}}{A_{1}^{r}+A_{2}^{r}+A_{3}^{r}+A_{4}^{r}},
\end{aligned}
$$

em que

$$
\begin{aligned}
& A_{1}=q_{x} \cosh (\beta J)+q_{y} \cosh (\beta D)+m_{x} \sinh (\beta J)+m_{y} \sinh (\beta D), \\
& A_{2}=q_{x} \cosh (\beta D)+q_{y} \cosh (\beta J)-m_{x} \sinh (\beta D)+m_{y} \sinh (\beta J), \\
& A_{3}=q_{x} \cosh (\beta D)+q_{y} \cosh (\beta J)-m_{x} \sinh (\beta J)-m_{y} \sinh (\beta D)
\end{aligned}
$$

$\mathrm{e}$

$$
A_{4}=q_{x} \cosh (\beta D)+q_{y} \cosh (\beta J)+m_{x} \sinh (\beta D)-m_{y} \sinh (\beta J) .
$$

\subsubsection{Limite de coordenação infinita}

Embora o problema já esteja formulado e seja perfeitamente possível analisar as relações de recorrência (2.52) a (2.54), é interessante recorrer a um limite de coordenação infinita, que deve produzir soluções correspondentes ao cálculo de campo médio,

O limite de coordenação infinita é dado por $r \rightarrow \infty ; J_{1}, J_{2} \rightarrow 0$, com $J_{1} r$ e $J_{2} r$ fixos. Nesse limite obtemos

$$
\begin{array}{ll}
A_{1}^{r} \rightarrow e^{\beta J r m_{x}+\beta D r m_{y}} ; & A_{2}^{r} \rightarrow e^{-\beta D r m_{x}+\beta J r m_{y}} ; \\
A_{3}^{r} \rightarrow e^{-\beta J r m_{x}-\beta D r m_{y}} ; & A_{4}^{r} \rightarrow e^{\beta D r m_{x}-\beta J r m_{y}} .
\end{array}
$$

Assim, lembrando que $q_{y}=1-q_{x}$, nesse limite de coordenação infinita o problema fica mais simples, reduzido a duas relações de recorrência apenas,

$$
\begin{aligned}
& m_{x}^{\prime}=\frac{\sinh \left(\beta J r m_{x}+\beta \operatorname{Drm}_{y}\right)}{\cosh \left(\beta J r m_{x}+\beta D r m_{y}\right)+\cosh \left(-\beta \operatorname{Drm}_{x}+\beta J r m_{y}\right)} \\
& m_{y}^{\prime}=\frac{\sinh \left(-\beta D r m_{x}+\beta J r m_{y}\right)}{\cosh \left(\beta J r m_{x}+\beta D r m_{y}\right)+\cosh \left(-\beta D r m_{x}+\beta J r m_{y}\right)} .
\end{aligned}
$$

\subsubsection{Ponto fixo paramagnético}

A partir das relações (2.61) e (2.62), é fácil perceber que existe sempre um ponto fixo desordenado (paramagnético), com $m_{x}^{*}=0$ e $m_{y}^{*}=0$.

Linearizando essas relações nas vizinhanças desse ponto fixo trivial, obtemos

$$
\left(\begin{array}{l}
m_{x}^{\prime} \\
m_{y}^{\prime}
\end{array}\right) \approx \frac{1}{2}\left(\begin{array}{cc}
\beta J r & \beta D r \\
-\beta D r & \beta J r
\end{array}\right)\left(\begin{array}{l}
m_{x} \\
m_{y}
\end{array}\right) .
$$


Portanto, esse ponto fixo será linearmente estável para

$$
\frac{1}{2}|\beta J r \pm i \beta D r|<1
$$

Para fazer contato com a literatura, é conveniente escrever as variáveis $J$ e $D$ em termos do parâmetro adimensional $p=D / J$, que indica a relevância da quiralidade, e da temperatura adimensional,

$$
\frac{1}{T}=\beta J r
$$

Num diagrama da temperatura $T$ contra o parâmetro $p$, a região de estabilidade do ponto fixo trivial é dada por

$$
T>\frac{1}{2}\left(1+p^{2}\right)^{1 / 2}
$$

Na figura 4 exibimos essa região além de expor outros resultados que serão explicados adiante.

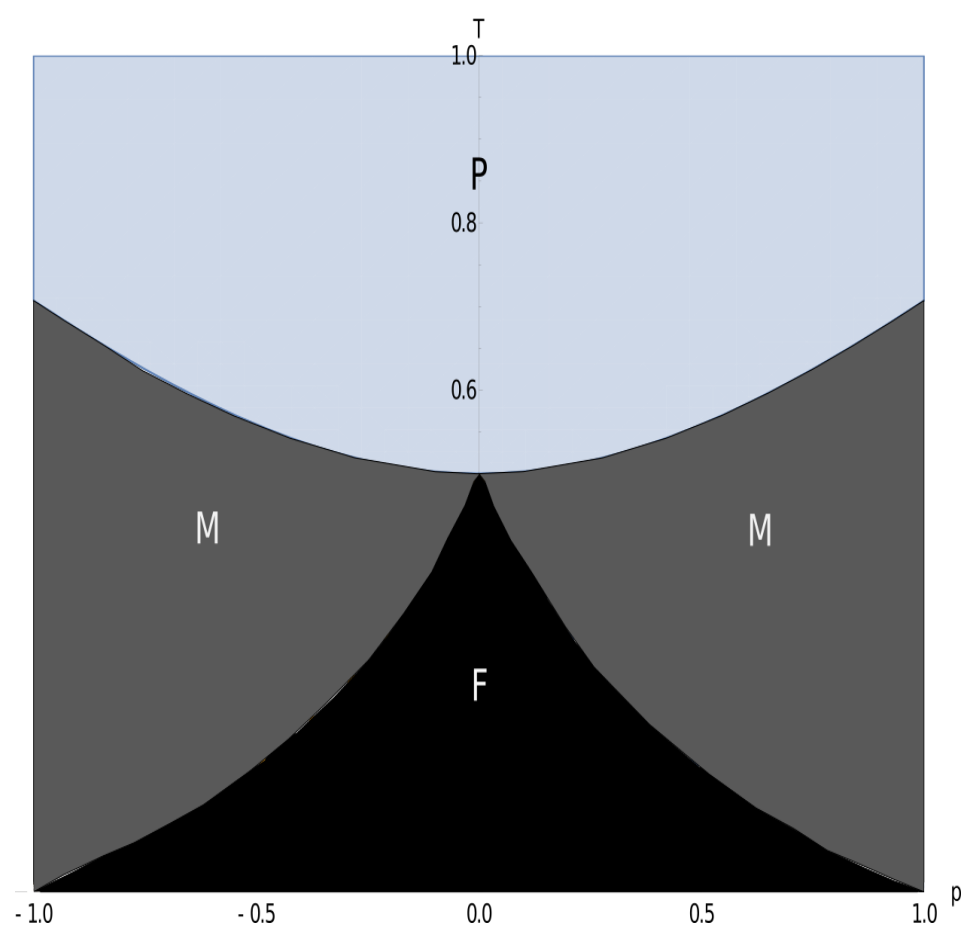

Figura 2.4: Diagrama de T (temperatura adimensional) contra o parâmetro $p$. A fase paramagnética se encontra na região chamada de $\mathrm{P}$, acima da linha que determina sua condição de estabilidade. As fases moduladas estão representadas nas regiões cinzas e a ferromagnética na região preta. 


\subsubsection{Cálculos numéricos na árvore de Cayley}

Para ir além da análise de estabilidade do ponto fixo paramagnético precisamos estudar numericamente as relações de recorrência (2.61) e (2.62). Dados $T$ e $p$, bem como os valores iniciais de $m_{x}$ e $m_{y}$, procuramos os atratores do mapeamento realizando um número muito grande (da ordem de 10 mil) iterações.

No caso desse modelo, não estamos preocupados com uma análise detalhada do diagrama de fases. Realizamos apenas um trabalho numérico exploratório, que é suficiente para fornecer indicações sobre a presença de muitas sequências de estruturas moduladas no interior da região ordenada do diagrama de fases. Para este modelo não temos interesse em estudar mais detalhadamente a fase modulada devido à literatura já existente[12].

As figuras a seguir mostram atratores encontrados dentro das regiões moduladas. Nessas figuras representamos o valor da magnetização no eixo y de spin contra a magnetizaçõ no eixo x de spin. Esses gráficos mostram mais de 10 mil iterações das equações de recorrência, depois de terem sido descartadas 10 mil iniciais normalmente, tendo com condições iniciais $m_{x}=$ $m_{y}=1$. Como pode ser visto os mesmos pontos se repetiram por essas 10 mil iterações.

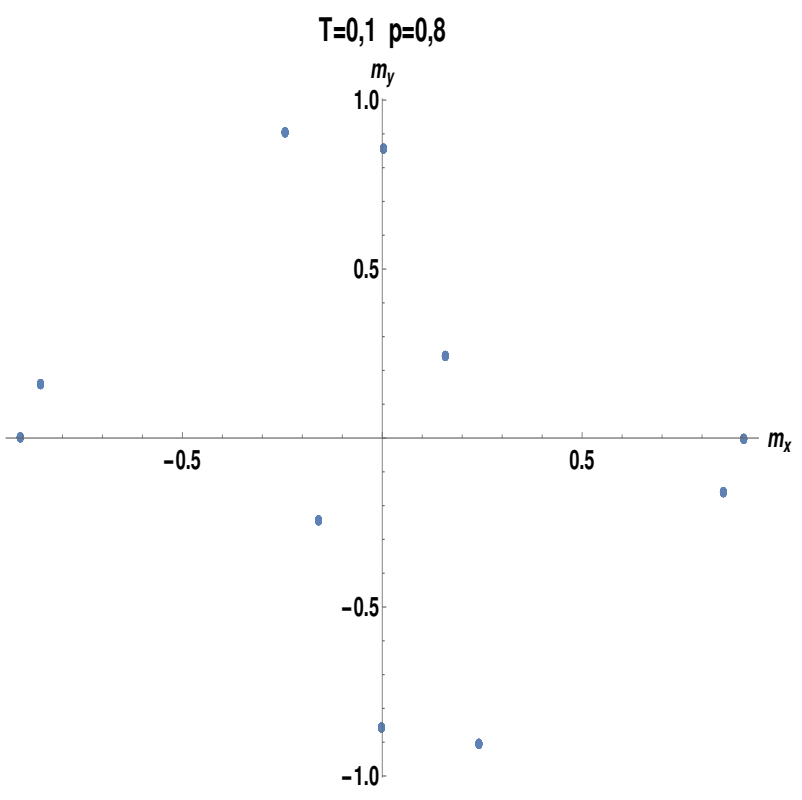

Figura 2.5: Atrator da magnetização para temperatura adimensional $T=0,1$ e parâmetro $p=D / J=0,8$. 


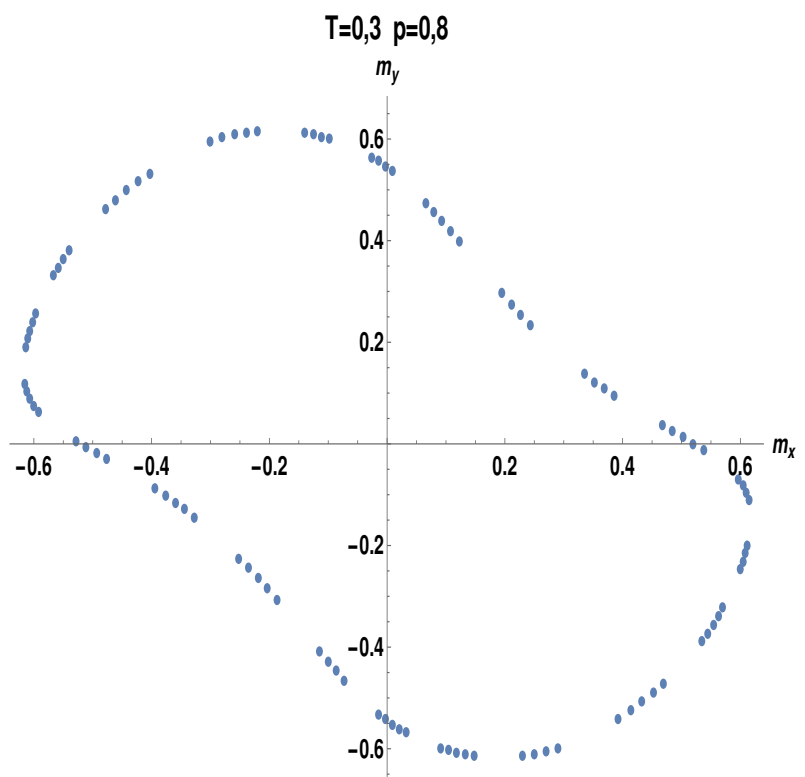

Figura 2.6: Atrator da magnetização para temperatura adimensional $T=0,3$ e parâmetro $p=D / J=0,8$.

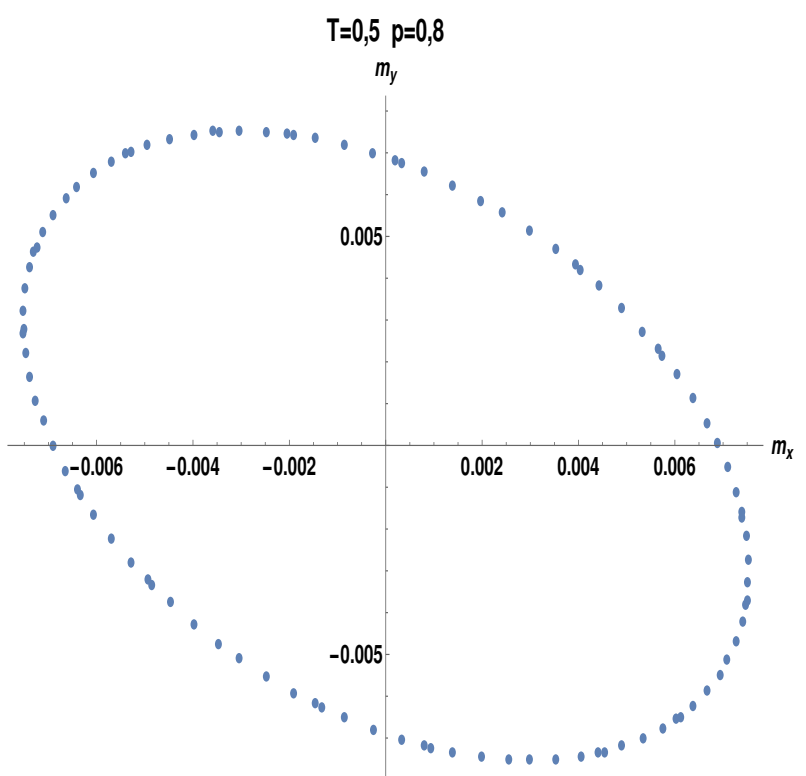

Figura 2.7: Atrator da magnetização para temperatura adimensional $T=0,5$ e parâmetro $p=D / J=0,8$.

É possível notar que com o aumento da temperatura a modulação tem um período cada vez maior. Além disso, o módulo dos valores alcançados da magnetização também diminui. Isso é uma indicação de que a transição entre a fase modulada e a paramagnética tem uma natureza de segunda ordem. 


\subsection{Modelo DM4 - solução de campo médio}

Consideramos agora uma solução convencional de campo médio do modelo DM4, definido numa rede cúbica simples, com o eixo de quiralidade ao longo da direção z. Aproximações desse tipo foram utilizadas de forma pioneira para analisar o diagrama de fases do modelo ANNNI e posteriormente para obter a estrutura de fases dos modelos quirais do relógio [4] [16] [17] [18].

Utilizando uma notação mais explícita para designar os sítios de uma rede cúbica simples, escrevemos

$$
\mathcal{H}_{4 C M}=\mathcal{H}_{1}+\mathcal{H}_{2}
$$

com

$$
\mathcal{H}_{1}=-J_{0} \sum_{z=1}^{N} \sum_{\left(x, y ; x^{\prime} y^{\prime}\right)}\left[S_{x, y, z}^{x} S_{x^{\prime}, y^{\prime}, z}^{x}+S_{x, y, z}^{y} S_{x^{\prime}, y \prime}^{y}, z\right],
$$

em que $\left(x, y ; x^{\prime} y^{\prime}\right)$ designa um par de vizinhos mais próximos num plano normal à direção $z, \mathrm{e}$

$$
\begin{aligned}
\mathcal{H}_{2} & =-J \sum_{x, y} \sum_{z=1}^{N}\left[S_{x, y, z}^{x} S_{x, y, z+1}^{x}+S_{x, y, z}^{y} S_{x, y, z+1}^{y}\right]- \\
& -D \sum_{x, y} \sum_{z=1}^{N}\left[S_{x, y, z}^{x} S_{x, y, z+1}^{y}-S_{x, y, z}^{y} S_{x, y, z+1}^{x}\right]
\end{aligned}
$$

em que a primeira soma (sobre $x$ e $y$ ) é sobre todos os $N^{2}$ sítios do plano $(x, y)$, e a segunda soma (sobre $z$ ) leva em conta as interações de primeiros vizinhos ao longo da direção $z$.

Vamos então recorrer à "desigualdade de Bogoliubov",

$$
G(\mathcal{H}) \leq G_{0}\left(\mathcal{H}_{0}\right)+\left\langle\mathcal{H}-\mathcal{H}_{0}\right\rangle_{0}=\Phi
$$

em que $G(\mathcal{H})$ é a energia livre associada ao hamiltoniano $\mathcal{H}, G_{0}\left(\mathcal{H}_{0}\right)$ é a energia livre associada a um hamiltoniano de tentativa $\mathcal{H}_{0}$, e $\langle\ldots\rangle_{0}$ é um valor esperado obtido num ensemble definido pelo hamiltoniano de tentativa. Com o objetivo de preservar a simetria das soluções, vamos escolher um hamiltoniano de tentativa por camadas, dado por

$$
\mathcal{H}_{0}=-\sum_{x, y} \sum_{z} \vec{\eta}_{z} \cdot \vec{S}_{x, y, z}=-\sum_{x, y} \sum_{z}\left[\eta_{z}^{x} S_{x, y, z}^{x}+\eta_{z}^{y} S_{x, y, z}^{y}\right]
$$

em que $\vec{\eta}_{z}=\left(\eta_{z}^{x}, \eta_{z}^{y}\right)$ é um campo de tentativa bidimensional. A solução de campo médio é obtida pela minimização da energia livre de tentativa $\Phi$ em relação a $\left\{\vec{\eta}_{z}\right\}$. 
Não é difícil mostrar que

$$
G_{0}=-\frac{N^{2}}{\beta} \sum_{z} \ln \left[2 \cosh \left(\beta \eta_{z}^{x}\right)+2 \cosh \left(\beta \eta_{z}^{y}\right)\right]
$$

e que

$$
\begin{gathered}
\frac{1}{N^{2}}\left\langle\mathcal{H}-\mathcal{H}_{0}\right\rangle_{0}=-2 J_{0} \sum_{z}\left[\left(m_{z}^{x}\right)^{2}+\left(m_{z}^{y}\right)^{2}\right]-J \sum_{z}\left[m_{z}^{x} m_{z+1}^{x}+m_{z}^{y} m_{z+1}^{y}\right]- \\
-D \sum_{z}\left[m_{z}^{x} m_{z+1}^{y}-m_{z}^{y} m_{z+1}^{x}\right]+\sum_{z}\left[\eta_{z}^{x} m_{z}^{x}+\eta_{z}^{y} m_{z}^{y}\right]
\end{gathered}
$$

em que usamos as definições

$$
m_{z}^{x}=\frac{\sinh \left(\beta \eta_{z}^{x}\right)}{\cosh \left(\beta \eta_{z}^{x}\right)+\cosh \left(\beta \eta_{z}^{y}\right)}
$$

$\mathrm{e}$

$$
m_{z}^{y}=\frac{\sinh \left(\beta \eta_{z}^{y}\right)}{\cosh \left(\beta \eta_{z}^{x}\right)+\cosh \left(\beta \eta_{z}^{y}\right)} .
$$

Agora podemos utilizar as definições dadas pelas equações (2.74) e (2.75) para escrever $\Phi$ na forma de uma série de potências nas magnetizações, fazendo contato com uma expansão do tipo Landau-Ginzburg Mantendo apenas termos até segunda ordem nas magnetizações, é fácil mostrar que

$$
\begin{gathered}
\frac{1}{N^{2}} \Phi=-\frac{1}{\beta} 2 N \ln 2+\sum_{z}\left(-2 J_{0}+\frac{1}{\beta}\right)\left[\left(m_{z}^{x}\right)^{2}+\left(m_{z}^{y}\right)^{2}\right]- \\
-J \sum_{z}\left[m_{z}^{x} m_{z+1}^{x}+m_{z}^{y} m_{z+1}^{y}\right]-D \sum_{z}\left[m_{z}^{x} m_{z+1}^{y}-m_{z}^{y} m_{z+1}^{x}\right]+\cdots
\end{gathered}
$$

Para diagonalizar essa forma quadrática, recorremos a uma representação de Fourier. Escrevemos então

$$
m_{z}^{x}=\frac{1}{\sqrt{N}} \sum_{q} m_{q}^{x} \exp (i q z) ; \quad m_{z}^{y}=\frac{1}{\sqrt{N}} \sum_{q} m_{q}^{y} \exp (i q z)
$$

com as somas restritas à primeira zona de Brillouin. Também é interessante escrever $m_{q}^{x}$ e $m_{q}^{y}$ em termos de suas partes reais e imaginárias,

$$
m_{q}^{x}=\frac{1}{\sqrt{2}}\left(R_{q}^{x}+i I_{q}^{x}\right), \quad m_{q}^{y}=\frac{1}{\sqrt{2}}\left(R_{q}^{y}+i I_{q}^{y}\right)
$$

Levando em conta que as magnetizações são reais, e que a zona de Brillouin é simétrica, também temos

$$
R_{q}=R_{-q}, \quad I_{q}=-I_{-q}
$$


para as componentes $x$ ou $y$. Então não é difícil mostrar que (ignorando constantes de normalização)

$$
\begin{gathered}
\frac{1}{N^{2}} \Phi=-\frac{1}{\beta} 2 N \ln 2+\sum_{q}\left(\frac{1}{\beta}-2 J_{0}-J \cos q\right)\left[\left(R_{q}^{x}\right)^{2}+\left(I_{q}^{x}\right)^{2}+\left(R_{q}^{y}\right)^{2}+\left(I_{q}^{y}\right)^{2}\right]- \\
-2 D \sum_{q}(\operatorname{sen} q)\left[I_{q}^{x} R_{q}^{y}-I_{q}^{y} R_{q}^{x}\right]+\cdots
\end{gathered}
$$

Portanto, até termos de segunda ordem, a energia livre de tentativa fica expressa em termos de modos reais e independentes no espaço de Fourier, mas acoplados nos planos $x-y$. Vamos agora definir as grandezas

$$
A=\frac{1}{\beta}-2 J_{0}-J \cos q ; \quad B=2 D \operatorname{sen} q,
$$

e utilizar a notação simplificada

$$
x_{1}=R_{q}^{x} ; \quad x_{2}=I_{q}^{x} ; \quad y_{1}=R_{q}^{y} ; \quad y_{2}=I_{q}^{y}
$$

Portanto, temos que analisar a forma quadrática

$$
Q=A\left(x_{1}^{2}+x_{2}^{2}+y_{1}^{2}+y_{2}^{2}\right)+B\left(y_{2} x_{1}-x_{2} y_{1}\right)=(\vec{v})^{t} \mathbf{M}(\vec{v})
$$

em que $\vec{v}$ é o vetor coluna $\left(x_{1}, x_{2}, y_{1}, y_{2}\right)$ e $\mathbf{M}$ é uma matriz supersimétrica,

$$
\mathbf{M}=\left(\begin{array}{cccc}
A & 0 & 0 & B / 2 \\
0 & A & -B / 2 & 0 \\
0 & -B / 20 & A & 0 \\
B / 2 & 0 & 0 & A
\end{array}\right)
$$

com autovalores degenerados,

$$
\lambda=A \pm \frac{1}{2} B=\frac{1}{\beta}-2 J_{0}-J \cos q \pm D \text { sen } .
$$

Portanto, a temperatura crítica da fase paramagnética é dada por

$$
\frac{1}{\beta_{c}}=k_{B} T_{c}=\max _{q}\left[2 J_{0}+J \cos q \pm D \operatorname{sen} q\right] .
$$

Para $D=0$, que é o caso do ferromagneto simples, a temperatura crítica é conhecida,

$$
\frac{1}{\beta_{c}}=k_{B} T_{c}=\max _{q}\left(2 J_{0}+J \cos q\right)=2 J_{0}+J
$$

pois $q_{c}=0$ é o primeiro valor do número de onda associado a um autovalor nulo da forma quadrática. 
Para $D \neq 0$, a maximação em relação ao número de onda fornece dois resultados

$$
\tan q= \pm \frac{D}{J}
$$

que correspondem a ondas propagantes nos dois sentidos do eixo $z$, com uma temperatura crítica dada por

$$
\frac{1}{\beta_{c}}=k_{B} T_{c}=2 J_{0}+\left(J^{2}+D^{2}\right)^{1 / 2}
$$

que é o resultado análogo da expressão no limite de coordenação infinita, obtido para o mesmo tipo de modelo definido na árvore de Cayley. Os dois métodos se diferenciam em seus resultados pelo termo constante extra que não existe para a árvore de Cayley. 


\section{Capítulo 3}

\section{Modelo de seis estados (DM6)}

Novamente consideramos um ferromagneto de Heisenberg com a adição de interações quirais, do tipo Dzyaloshinskii-Moriya. Na sua forma mais simples, o hamiltoniano de spin desse sistema é dado por

$$
\mathcal{H}=-J \sum_{\left(\vec{r}, \vec{r}^{\prime}\right)} \vec{S}_{\vec{r}} \cdot \vec{S}_{\vec{r}^{\prime}}-D \sum_{\left(\vec{r}, \vec{r}^{\prime}\right)}\left(\vec{S}_{\vec{r}} \times \vec{S}_{\vec{r}^{\prime}}\right) \cdot \hat{z}
$$

em que $\vec{S} \vec{r}$ é um "vetor de spin" no sítio $\vec{r}$ da rede cristalina, a primeira soma é sobre pares de sítios vizinhos mais próximos, e a segunda soma é sobre os vizinhos mais próximos ao longo da direção $z$ (estamos considerando um sistema monoaxial, com interações quirais ao longo do eixo $z)$.

Vamos agora supor que as variáveis de spin podem assumir seis estados vetoriais, ao longo das direções dos eixos cartesianos. Temos assim os "estados de spin"

$$
\vec{S}=\left(\begin{array}{l}
1 \\
0 \\
0
\end{array}\right),\left(\begin{array}{l}
0 \\
1 \\
0
\end{array}\right),\left(\begin{array}{l}
0 \\
0 \\
1
\end{array}\right),\left(\begin{array}{c}
-1 \\
0 \\
0
\end{array}\right),\left(\begin{array}{c}
0 \\
-1 \\
0
\end{array}\right),\left(\begin{array}{c}
0 \\
0 \\
-1
\end{array}\right)
$$

Nessas circunstâncias é definido o modelo DM6, um modelo de seis estados ao longo de três eixos, mais distante dos modelos do relógio, mas mais próximo de uma versão quiral do modelo clássico de Heisenberg com a inclusão de um termo do tipo DM.

Na mesma linha do estudo apresentada para o DM4 percorremos as seguintes etapas:

1. Inicialmente utilizamos a técnica da matriz de transferência para analisar exatamente uma versão unidimensional do hamiltoniano associado ao modelo DM6. Novamente, com parâmetros positivos, $J>0$ e $D>0$, mostramos que o estado fundamental é ferromagnético para 
$p=D / J<1 / 2$. Há um ponto de multifase para $p=1 / 2$. Acima desse valor há uma estrutura espacialmente modulada, como será identificado logo adiante.

2. Em seguida, formulamos o análogo desse problema numa árvore de Cayley. Nessas condições, sem maiores dificuldades, além de resultados analíticos são obtidos resultados numéricos para aspectos sutis do diagrama de fases. Em particular, recorremos ao limite de coordenação infinita da árvore de Cayley, em que as expressões algébricas ficam mais simples, com a expectativa de obter resultados em concordância qualitativa com cálculos usuais de campo médio para o modelo análogo numa rede de Bravais. Apontamos a existência de fases espacialmente moduladas, como ocorre no caso do modelo DM4.

3. Finalmente apresentamos uma solução de campo médio, com base na desigualdade de Bogoliubov, que deve exibir as mesmas características qualitativas da solução exata no limite de coordenação infinita da árvore de Cayley. Obtivemos o termo dominante (quadrático) de uma expansão de Landau, fazendo contato com resultados fenomenológicos da literatura. No futuro pretendemos analisar mais detalhadamente essa solução de campo médio

\subsection{Modelo DM6 em uma dimensão}

Em uma dimensão o hamiltoniano de spin do modelo DM6 é dado por

$$
\mathcal{H}_{1 D M 6}=-J \sum_{i=1}^{N} \vec{S}_{i} \cdot \vec{S}_{i+1}-D \sum_{i=1}^{N}\left(\vec{S}_{i} \times \vec{S}_{i+1}\right) \cdot \hat{z}
$$

com os estados de spin dados pela equação (3.2).

Usando o formalismo convencional do ensemble canônico, obtemos a matriz de transferência

em que

$$
\mathbf{T}_{6}=\left(\begin{array}{cccccc}
K & \Delta & 1 & K^{-1} & \Delta^{-1} & 1 \\
\Delta^{-1} & K & 1 & \Delta & K^{-1} & 1 \\
1 & 1 & K & 1 & 1 & K^{-1} \\
K^{-1} & \Delta^{-1} & 1 & K & \Delta & 1 \\
\Delta & K^{-1} & 1 & \Delta^{-1} & K & 1 \\
1 & 1 & K^{-1} & 1 & 1 & K
\end{array}\right)
$$

$$
K=\exp (\beta J) ; \quad \Delta=\exp (\beta D) .
$$

Notando a estrutura em blocos $3 \times 3$ dessa matriz, podemos mudar para uma representação em 
que ela se torna blocodiagonal. Vamos então escrever a matriz de inversão dos blocos,

$$
\mathbf{B}_{6}=\left(\begin{array}{llllll}
0 & 0 & 0 & 1 & 0 & 0 \\
0 & 0 & 0 & 0 & 1 & 0 \\
0 & 0 & 0 & 0 & 0 & 1 \\
1 & 0 & 0 & 0 & 0 & 0 \\
0 & 1 & 0 & 0 & 0 & 0 \\
0 & 0 & 1 & 0 & 0 & 0
\end{array}\right)
$$

que pode ser diagonalizada pela transformação ortogonal

$$
\mathbf{U}=\frac{1}{\sqrt{2}}\left(\begin{array}{cccccc}
1 & 0 & 0 & -1 & 0 & 0 \\
0 & 1 & 0 & 0 & -1 & 0 \\
0 & 0 & 1 & 0 & 0 & -1 \\
1 & 0 & 0 & 1 & 0 & 0 \\
0 & 1 & 0 & 0 & 1 & 0 \\
0 & 0 & 1 & 0 & 0 & 1
\end{array}\right)
$$

Temos então a matriz $\mathbf{T}_{6}$ transformada,

$$
\begin{aligned}
& \mathbf{T}_{6}^{\prime}=\mathbf{U} \mathbf{T}_{6} \widetilde{\mathbf{U}}= \\
& =2\left(\begin{array}{cccccc}
\sinh \beta J & \sinh \beta D & 0 & 0 & 0 & 0 \\
-\sinh \beta D & \sinh \beta J & 0 & 0 & 0 & 0 \\
0 & 0 & \sinh \beta J & 0 & 0 & 0 \\
0 & 0 & 0 & \cosh \beta J & \cosh \beta D & 1 \\
0 & 0 & 0 & \cosh \beta D & \cosh \beta J & 1 \\
0 & 0 & 0 & 1 & 1 & \cosh \beta J
\end{array}\right) \text {, }
\end{aligned}
$$

de onde obtemos os seis autovalores,

$$
\begin{gathered}
\lambda_{1}=2 \sinh \beta J, \\
\lambda_{2,3}=2 \sinh \beta J \pm i 2 \sinh \beta D, \\
\lambda_{4}=2 \cosh \beta J-2 \cosh \beta D,
\end{gathered}
$$

$\mathrm{e}$

$$
\lambda_{5,6}=2 \cosh \beta J+\cosh \beta D \pm \sqrt{\cosh ^{2} \beta D+8} .
$$


Levando em conta que $J>0$ (isto é, $K>1$ ), vamos também tomar $D>0$ (ou seja, $\Delta>1$ ), e examinar o estado fundamental desse sistema. No limite de temperatura nula, temos

$$
\begin{gathered}
\lambda_{1} \rightarrow K ; \quad \lambda_{2,3} \rightarrow K \pm i \Delta ; \\
\lambda_{4} \rightarrow K-\Delta ; \quad \lambda_{5} \rightarrow \Delta+K ; \quad \lambda_{6} \rightarrow K .
\end{gathered}
$$

Então, com $D<J$, o termo dependente de $K$ sempre predomina. Nesse caso há uma degenerescência dos seis autovalores, que assumem valores reais, indicando um estado fundamental ferromagnético. Com $D>J$ também há uma degenerescência, mas de ordem quádrupla, com autovalores complexos conjugados, indicando um ordenamento espacialmente modulado, muito semelhante ao caso do modelo $D M 4$. Quando $D=J$, o maior autovalor em módulo é $\lambda_{5} \rightarrow 2 K$, indicando a existência de um ponto de multifase, associado à entropia residual $k_{B} \ln 2$.

Não é difícil mostrar que $\lambda_{5}$ é o maior autovalor para qualquer temperatura finita. De fato, basta perceber que $\lambda_{5}>\lambda_{6}$ ou $\lambda_{4}$, e que $\left|\lambda_{2,3}\right|>\lambda_{1}$. Aí é fácil verificar que $\lambda_{5}$ também é maior do que $\left|\lambda_{2,3}\right|$. Então, no limite termodinâmico, a função canônica de partição desse sistema, $Z_{D M 6}$, é dada por

$$
\frac{1}{N} \ln Z_{D M 6}=\ln \lambda_{5}
$$

A partir dessa função canônica, podemos obter expressões para a energia interna e a entropia por sítio, que estão representadas nas figuras 1 e 2, para temperaturas suficientemente baixas (e que estão de acordo com a nossa análise qualitativa do estado fundamental). 


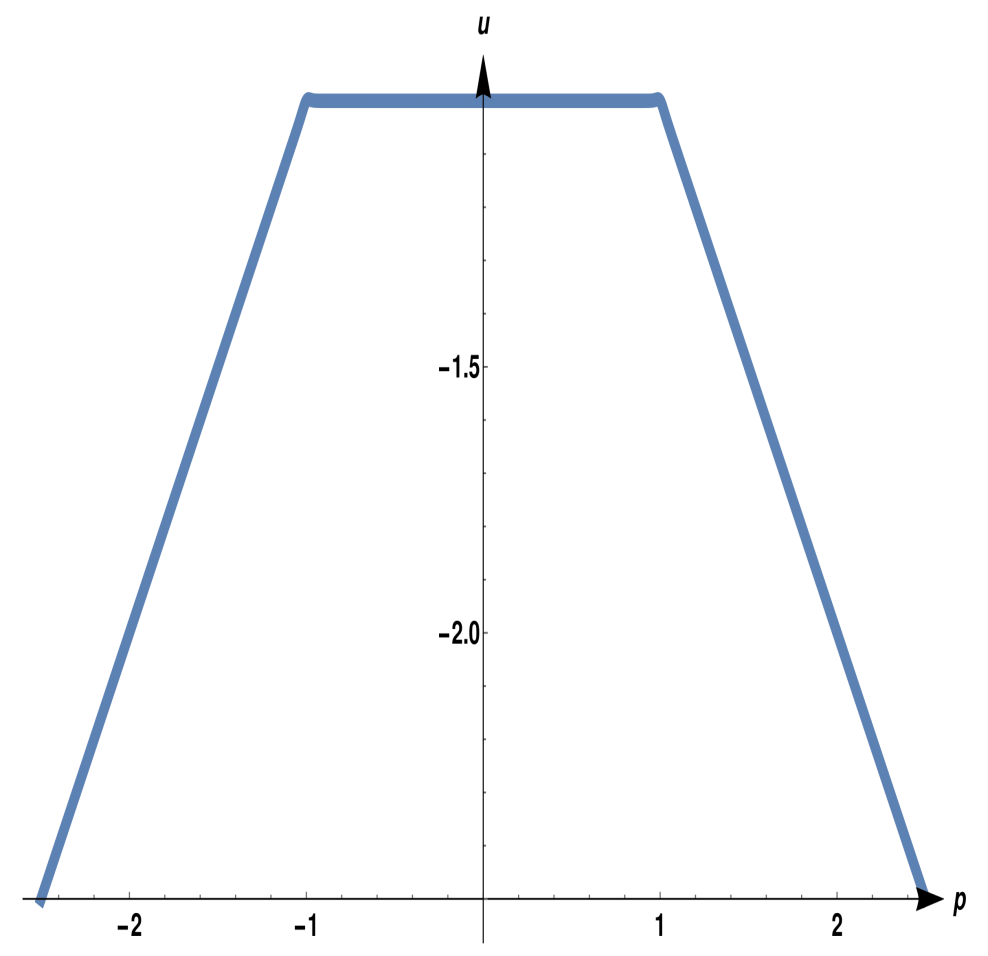

Figura 3.1: Energia interna $u$ por sítio, em unidades do parâmetro de troca $J>0$, contra $p=D / J$, para temperaturas suficientemente baixas. 


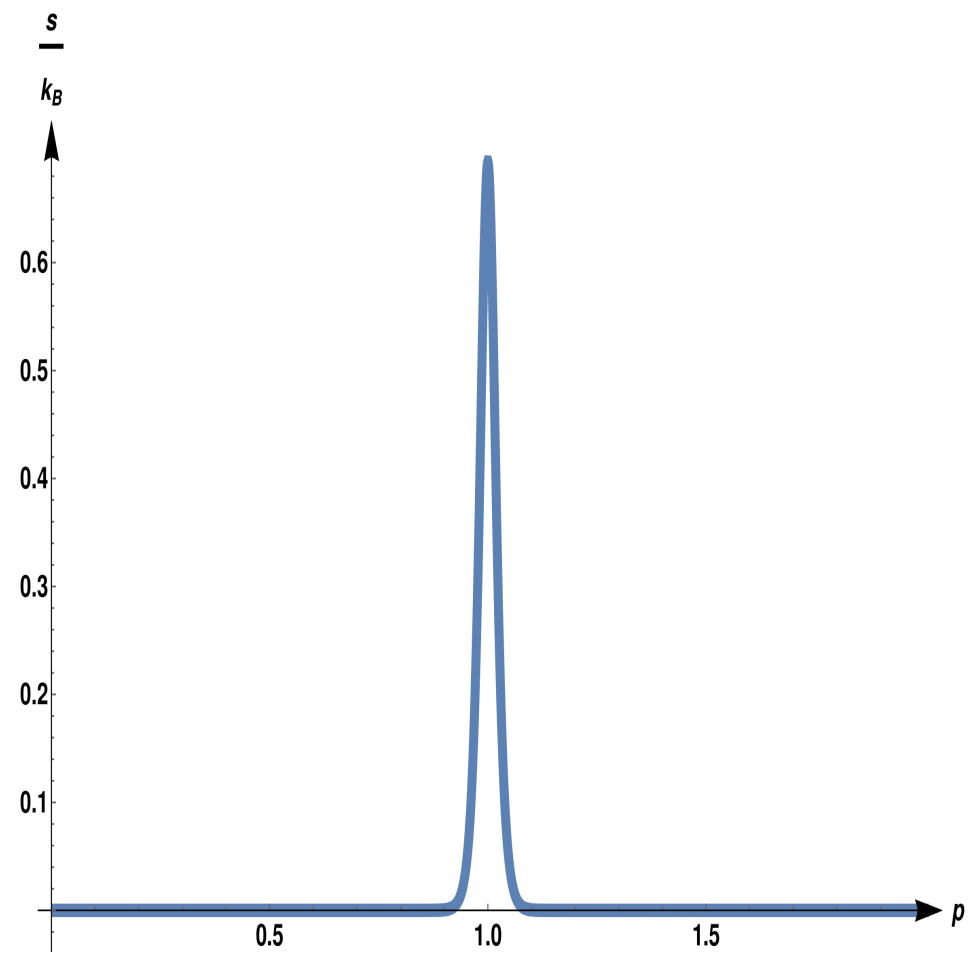

Figura 3.2: Entropia por partícula $s$, em unidades da constante de Boltzmann $k_{B}$, contra o parâmetro $p=D / J$, para temperaturas suficientemente baixas. O crescimento da entropia nas vizinhanças de $p=1$ indica a existência de um ponto de multifase (com entropia residual, de acordo com a nossa análise do estado fundamental).

Também é simples obter de forma explícita os limites da energia $u$ e da entropia $s$ para temperaturas muito baixas. Para $|D| \leq J$, temos

$$
\lim _{\beta \rightarrow \infty} u=-J
$$

Por outro lado, para $|D|>J$, temos

$$
\lim _{\beta \rightarrow \infty} u=D,
$$

que está de acordo com o gráfico da figura 3.1. Da mesma forma, um cálculo simples para a entropia indica o valor limite $s=0$, exceto para $D=J$, no ponto de multifase, em que obtemos a entropia residual $s=k_{B} \ln 2$, que é o mesmo valor obtido no caso do modelo $D M 4$.

\subsubsection{Estruturas de fases no estado fundamental}

Para identificar as estruturas de fase do estado fundamental devemos analisar a energia interna de cada arranjo de spins. Para $|D|>J$, devemos ter

$$
u \rightarrow-J\left(\vec{S}_{i} \cdot \vec{S}_{i+1}\right)-D\left(\vec{S}_{i} \times \vec{S}_{i+1}\right) . \hat{z} \rightarrow D
$$


Neste caso precisamos que o termo dependente de $D$ seja -1 e que o termo dependente de $J$ seja 0. Logo, temos estruturas de fase do tipo

$$
\{\ldots \leftarrow \uparrow \rightarrow \downarrow \leftarrow \uparrow \ldots\}
$$

que correspondem a um arranjo de natureza quiral.

Para $|D|<J$, a partir de considerações semelhantes, temos

$$
\vec{S}_{i}=\vec{S}_{i+1}
$$

Portanto, há uma fase ferromagnética seis vezes degenerada, de acordo com as nossas considerações sobre os autovalores da matriz de transferência. Temos então os seguintes arranjos de spins:

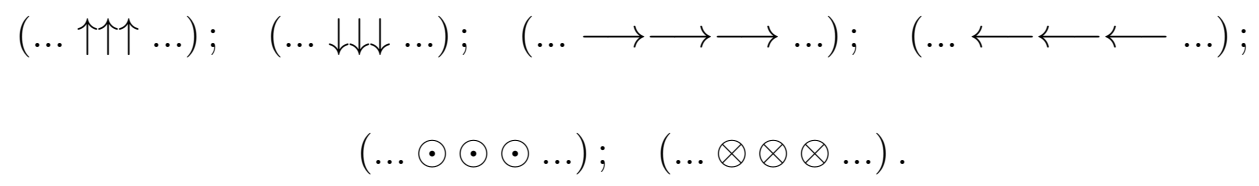

Para $|D|=J$, temos uma enorme variedade de combinações possíveis de fases, que acaba levando à entropia residual $s=k_{B} \ln 2$.

\subsection{Modelo DM6 na árvore de Cayley}

Formulamos o modelo ao longo dos ramos de uma árvore de Cayley, da mesma maneira como já fizemos no caso do modelo DM4. Assim obtivemos as seguintes relações de recorrência:

$$
\begin{aligned}
Z_{1}^{\prime}= & \left(e^{\beta J} Z_{1}+e^{\beta D} Z_{2}+Z_{3}+e^{-\beta J} Z_{4}+e^{-\beta D} Z_{5}+Z_{6}\right)^{r} ; \\
Z_{2}^{\prime}= & \left(e^{-\beta D} Z_{1}+e^{\beta J} Z_{2}+Z_{3}+e^{\beta D} Z_{4}+e^{-\beta J} Z_{5}+Z_{6}\right)^{r} ; \\
& Z_{3}^{\prime}=\left(Z_{1}+Z_{2}+e^{\beta J} Z_{3}+Z_{4}+Z_{5}+e^{-\beta J} Z_{6}\right)^{r} ; \\
Z_{4}^{\prime}= & \left(e^{-\beta J} Z_{1}+e^{-\beta D} Z_{2}+Z_{3}+e^{\beta J} Z_{4}+e^{\beta D} Z_{5}+Z_{6}\right)^{r} ; \\
Z_{5}^{\prime}= & \left(e^{\beta D} Z_{1}+e^{-\beta J} Z_{2}+Z_{3}+e^{-\beta D} Z_{4}+e^{\beta J} Z_{5}+Z_{6}\right)^{r} ; \\
& Z_{6}^{\prime}=\left(Z_{1}+Z_{2}+e^{-\beta J} Z_{3}+Z_{4}+Z_{5}+e^{\beta J} Z_{6}\right)^{r},
\end{aligned}
$$


em que $Z_{i}^{\prime}$ e $Z_{i}$, para $i=1, \ldots, 6$, são “funções de partição canônicas parciais", associadas a vértices situados em gerações imediatamente sucessivas da árvore.

Nesta parte dos cálculos é conveniente definir uma magnetização efetiva por partícula,

$$
\begin{gathered}
\vec{m}=\langle\vec{S}\rangle=\left(\begin{array}{l}
m_{x} \\
m_{y} \\
m_{z}
\end{array}\right)= \\
=\frac{\vec{S}(1) Z_{1}+\vec{S}(2) Z_{2}+\vec{S}(3) Z_{3}+\vec{S}(4) Z_{4}+\vec{S}(5) Z_{5}+\vec{S}(6) Z_{6}}{Z_{1}+Z_{2}+Z_{3}+Z_{4}+Z_{5}+Z_{6}},
\end{gathered}
$$

e uma grandeza $\vec{q}$, associada ao quadrado das variáveis de spin,

$$
\vec{q}=\left(\begin{array}{c}
\left\langle S_{x}^{2}\right\rangle \\
\left\langle S_{y}^{2}\right\rangle \\
\left\langle S_{z}^{2}\right\rangle
\end{array}\right)=\left(\begin{array}{c}
q_{x} \\
q_{y} \\
q_{z}
\end{array}\right) .
$$

Então temos

$$
m_{x}=\frac{Z_{1}-Z_{4}}{\Omega} ; \quad m_{y}=\frac{Z_{2}-Z_{5}}{\Omega} ; \quad m_{z}=\frac{Z_{3}-Z_{6}}{\Omega}
$$

e

$$
q_{x}=\frac{Z_{1}+Z_{4}}{\Omega} ; \quad q_{y}=\frac{Z_{2}+Z_{5}}{\Omega} ; \quad q_{z}=\frac{Z_{3}+Z_{6}}{\Omega},
$$

com

$$
\Omega=Z_{1}+Z_{2}+Z_{3}+Z_{4}+Z_{5}+Z_{6}
$$

Com algumas manipulações algébricas, também semelhantes ao que já foi feito no caso do modelo $D M 4$, foram obtidas as relações de recorrência em termos dessas novas variáveis,

$$
\begin{aligned}
& m_{x}^{\prime}=\frac{A^{r}-D^{r}}{A^{r}+B^{r}+C^{r}+D^{R}+E^{r}+F^{r}} ; \\
& m_{y}^{\prime}=\frac{B^{r}-E^{r}}{A^{r}+B^{r}+C^{r}+D^{R}+E^{r}+F^{r}} ; \\
& m_{z}^{\prime}=\frac{C^{r}-F^{r}}{A^{r}+B^{r}+C^{r}+D^{R}+E^{r}+F^{r}} ; \\
& q_{z}^{\prime}=\frac{C^{r}+F^{r}}{A^{r}+B^{r}+C^{r}+D^{R}+E^{r}+F^{r}},
\end{aligned}
$$

em que

$$
\begin{aligned}
& A^{r}=\left(q_{x} \cosh (\beta J)+m_{x} \sinh (\beta J)+q_{y} \cosh (\beta D)+m_{y} \sinh (\beta D)+q_{z}\right)^{r}, \\
& B^{r}=\left(q_{x} \cosh (\beta D)-m_{x} \sinh (\beta D)+q_{y} \cosh (\beta J)+m_{y} \sinh (\beta J)+q_{z}\right)^{r},
\end{aligned}
$$




$$
\begin{gathered}
C^{r}=\left(q_{x}+q_{y}+q_{z} \cosh (\beta J)+m_{z} \sinh (\beta J)\right)^{r}, \\
D^{r}=\left(q_{x} \cosh (\beta J)-m_{x} \sinh (\beta J)+q_{y} \cosh (\beta D)-m_{y} \sinh (\beta D)+q_{z}\right)^{r} \\
E^{r}=\left(q_{x} \cosh (\beta D)+m_{x} \sinh (\beta D)+q_{y} \cosh (\beta J)-m_{y} \sinh (\beta J)+q_{z}\right)^{r}, \\
F^{r}=\left(q_{x}+q_{y}+q_{z} \cosh (\beta J)-m_{z} \sinh (\beta J)\right)^{r} .
\end{gathered}
$$

Numa etapa seguinte, utilizamos o limite de coordenação infinita da árvore de Cayley $(r \rightarrow$ $\infty, J, D \rightarrow 0$ com $J r$ e $D r$ fixos). O sistema de equações acopladas ficou mais simples, reduzindo-se a três equações de recorrência apenas. Nesse limite, que corresponde a um tipo de aproximação de campo médio, temos

$$
\begin{gathered}
A^{r} \rightarrow \exp \left(\beta J r m_{x}+\beta D r m_{y}\right) ; \quad B^{r} \rightarrow \exp \left(-\beta \operatorname{Drm}_{x}+\beta J r m_{y}\right) ; \\
C^{r} \rightarrow \exp \left(\beta J r m_{z}\right) ; \quad D^{r} \rightarrow \exp \left(-\beta J r m_{x}-\beta D r m_{y}\right) ; \\
E^{r} \rightarrow \exp \left(\beta D r m_{x}-\beta J r m_{y}\right) ; \quad F^{r} \rightarrow \exp \left(-\beta J r m_{z}\right),
\end{gathered}
$$

de onde obtemos três relações de recorrência,

$$
\begin{gathered}
m_{x}^{\prime}=\frac{1}{M}\left[\sinh \left(\beta \operatorname{Jrm}_{x}+\beta \operatorname{Drm}_{y}\right)\right], \\
m_{y}^{\prime}=\frac{1}{M}\left[\sinh \left(-\beta \operatorname{Drm}_{x}+\beta J r m_{y}\right)\right], \\
m_{z}^{\prime}=\frac{1}{M}\left[\sinh \left(\beta J r m_{z}\right)\right],
\end{gathered}
$$

com o denominador comum

$$
M=\cosh \left(\beta J r m_{x}+\beta \operatorname{Drm}_{y}\right)+\cosh \left(-\beta \operatorname{Drm}_{x}+\beta J r m_{y}\right)+\cosh \left(\beta J r m_{z}\right) .
$$

\subsubsection{Ponto fixo trivial}

É imediato perceber que as relações de recorrência (3.44) a (3.46) têm um ponto fixo trivial, de natureza desordenada, dado por $m_{x}^{*}=m_{y}^{*}=m_{z}^{*}=0$. Linearizando as relações de recorrência nas vizinhanças desse ponto fixo, obtemos

$$
\left(\begin{array}{l}
m_{x}^{\prime} \\
m_{y}^{\prime} \\
m_{z}^{\prime}
\end{array}\right) \approx \frac{1}{3}\left(\begin{array}{ccc}
\beta J r & \beta D r & 0 \\
-\beta D r & \beta J r & 0 \\
0 & 0 & \beta J r
\end{array}\right)\left(\begin{array}{l}
m_{x} \\
m_{y} \\
m_{z}
\end{array}\right) .
$$


A matriz $3 \times 3$ associada a essa forma linear tem três autovalores distintos, dados por

$$
\begin{gathered}
\lambda_{1}=\frac{1}{3} \beta J r ; \\
\lambda_{2}=\frac{1}{3}(\beta J r+i \beta D r) ; \\
\lambda_{3}=\frac{1}{3}(\beta J r-i \beta D r) .
\end{gathered}
$$

Tomando $\beta J r=1 / T$ e $D / J=p$, obtemos as condições de estabilidade da fase desordenada num diagrama $T-p$,

$$
T>\frac{1}{3}
$$

$\mathrm{e}$

$$
T^{2}>\frac{1}{9+p^{2}},
$$

como está indicado na figura 3 (e que é muito semelhante ao diagrama correspondente para o modelo DM4).

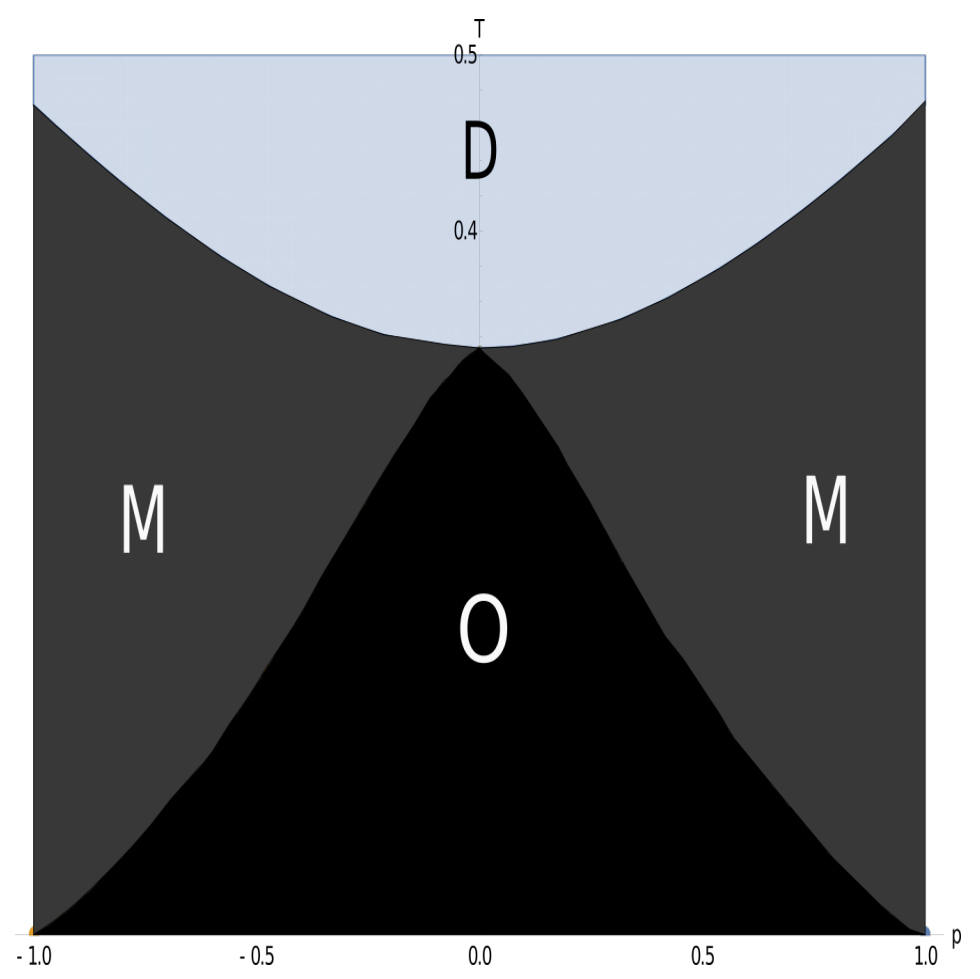

Figura 3.3: Diagrama da temperatura adimensional $T$ contra o parâmetro $p$. A fase desordenada se encontra na região "D", acima da linha que determina a sua condição de estabilidade. Fases espacialmente moduladas existem na regiões indicadas pela letra $M$, para qualquer $p \neq 0$. 
É interessante notar como evoluem as iterações desse sistema acoplado. A variável $m_{z}$ só depende da sua útima iteração para determinar o seu próximo valor. Então o sistema $m_{x}, m_{y}$ não é fortemente relacionado a $m_{z}$, nessa análise assintótica. A variável $m_{z}$ evolui, aproximadamente, ao longo de uma reta, enquanto o diagrama de $m_{y}$ versus $m_{x}$ apresenta oscilações semelhantes ao que acontece no caso do modelo de 4 estados.

\subsubsection{Regiões ordenadas do diagrama de fases}

Iterando as relações de recorrência (3.44) a (3.46), foi possível encontrar as fronteiras de transição entre as diferentes fases do sistema (desordenada, ordenada/ferromagneticamente, espacialmente moduladas). A modulação somente se manifesta para as componentes $x$ e $y$ dos spins (como é esperado a partir da análise assintótica). Através de manipulações das relações de recorrência, numa análise preliminar, observamos que a transição entre as fases ordenada e espacialmente moduladas deve ser de primeira ordem, enquanto a transição das fases moduladas para a desordenada é aparentemente contínua (de segunda ordem).

Também conseguimos verificar, pelo menos de forma qualitativa, comportamentos semelhantes a "escadas do diabo", ou seja, estruturas em que o número de onda das oscilações espaciais, para temperatura fixa, em função do parâmetro $p$, apresenta muitos patamares, de larguras aparentemente arbitrárias. As figuras 3.4-3.6 mostram esse tipo de comportamento. 


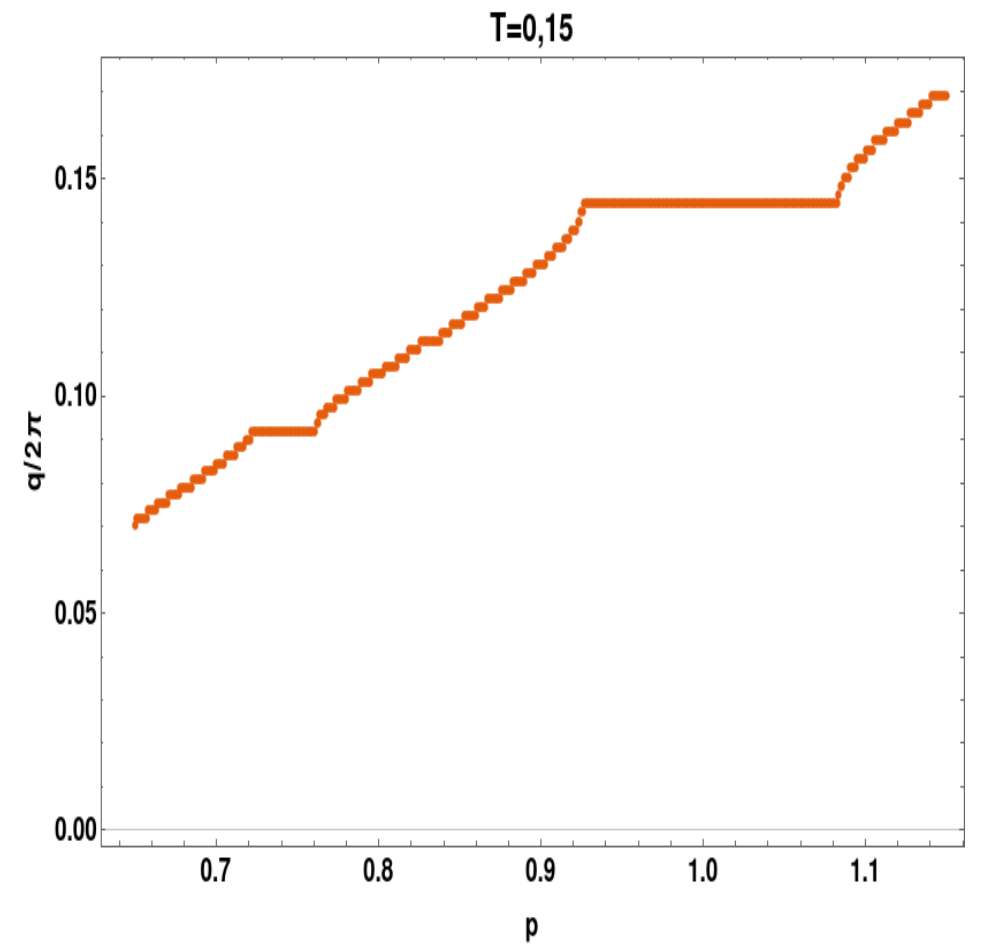

Figura 3.4: Frequência normalizada contra o parâmetro $p$ para temperatura fixa, $T=0,15$. Há forte indicação da presença de estruturas do tipo "escada do diabo".

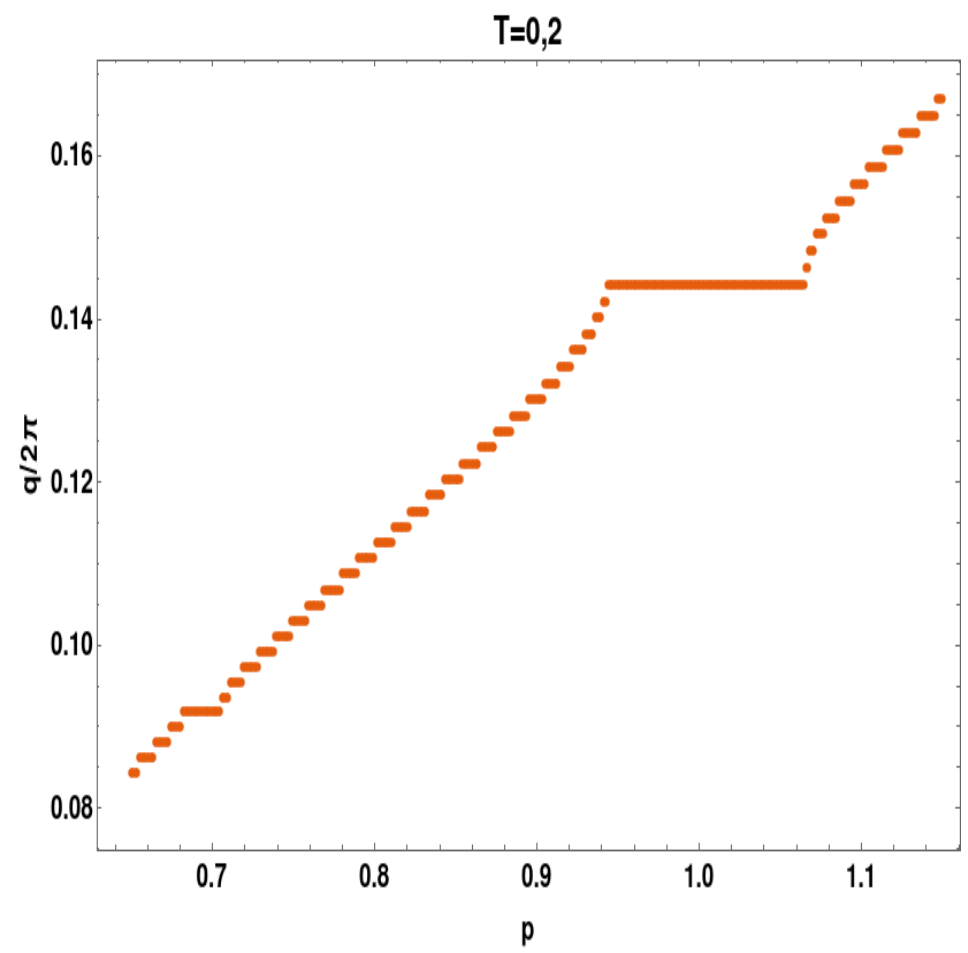

Figura 3.5: Frequência normalizada contra o parâmetro $p$ para temperatura $T=0,2$. 


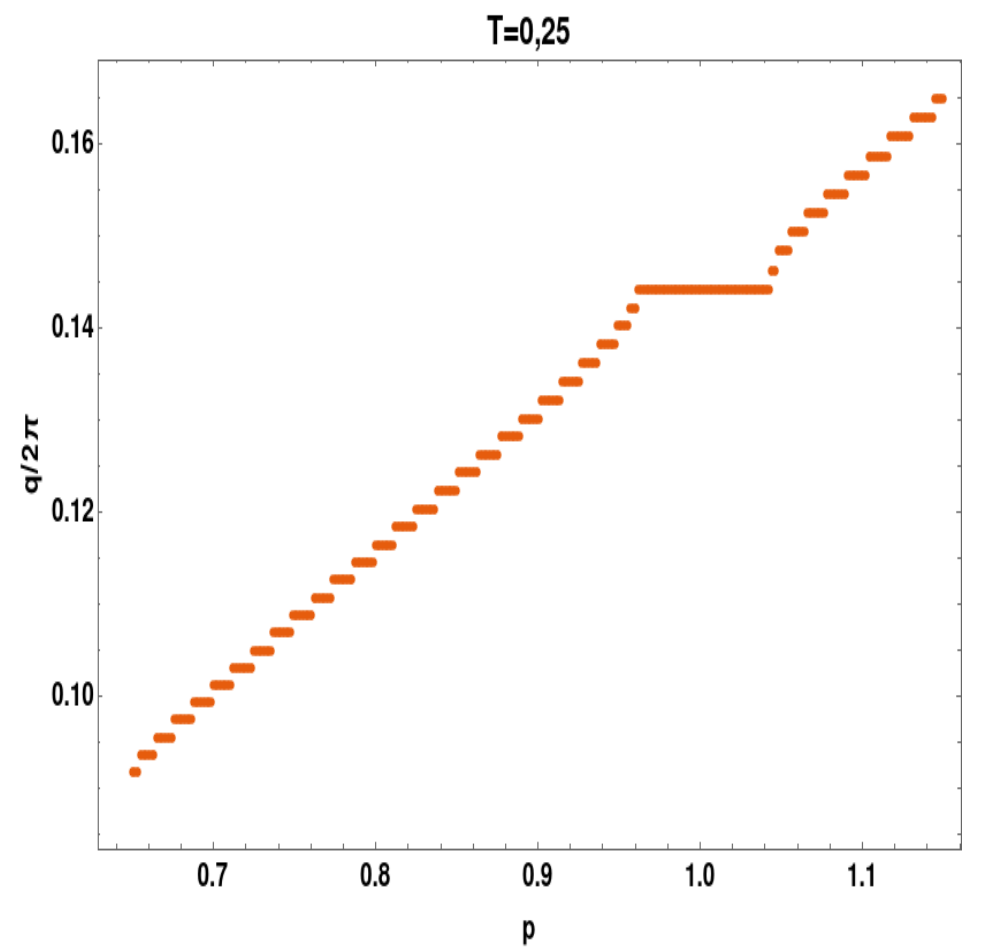

Figura 3.6: Frequência normalizada contra o parâmetro $p$ a temperatura $T=0,25$.

As frequências (harmônico principal na análise de Fourier) são obtidas fazendo uma analíse de Fourier num gráfico de $m_{x}$ ou $m_{y}$ por número de interações (isso é feito ápos o sistema sair so transiente, obviamente). Para obter essas figuras diversas condições inicias foram utilizadas, todas geram as mesmas imagens para os mesmos valores de $p$ e $T$.

\subsubsection{Medida da dimensão de Hausdorff}

Para obter uma caracterização mais precisa das fases moduladas e evidenciar a existência das escadas do diabo, investigamos a dimensão fractal dos patamares exibidos nas figuras anteriores.

Para obter a dimensão fractal de Hausdorff foi aplicado um método chamado "box-counting"[19]. Primeiramente foi tomado um valor $\epsilon>0$ e calculada a soma de todos os "degraus"cujo comprimento, ao longo do eixo $p$, é maior que $\epsilon$. Subtraindo esse valor do comprimeto total ao longo do eixo $p,\left(p_{f}-p_{i}\right)$, obtemos a grandeza $X(\epsilon)$, que é o comprimento restante no intervalo. Fazemos então um ajuste linear de $\log (X(\epsilon) / \epsilon)$ contra $\log (1 / \epsilon)$. Para valores de $\epsilon$ próximos de 0 a inclinação desse ajuste fornece uma boa aproximação do valor da dimensão fractal da imagem.

Para testar essa implementação do algoritmo, foi utilizada a função de Cantor. Esta função no domínio $[0,1]$ tem a mesma dimensão do conjunto de Cantor $\left(D_{H}=\log (2) / \log (3) \approx\right.$ 0,6309). Essa função se comporta exatamente como as "escadas do diabo"obtidas para o sis- 
tema estudado. Assim nosso algoritmo pode ser aplicado e testado diretamente [20]. As figuras a seguir (3.7 e 3.8) mostram a função de Cantor e o ajuste obtido para a sua dimensão.

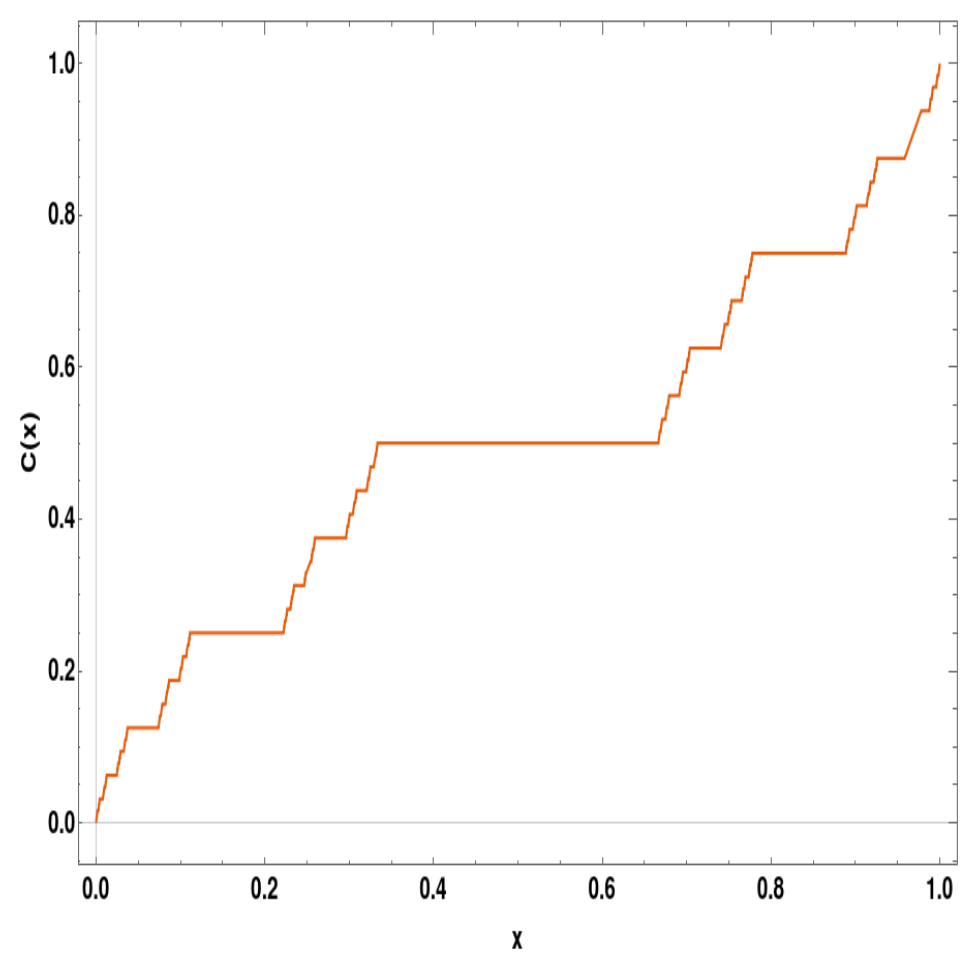

Figura 3.7: A função de Cantor no domínio [0,1]. 


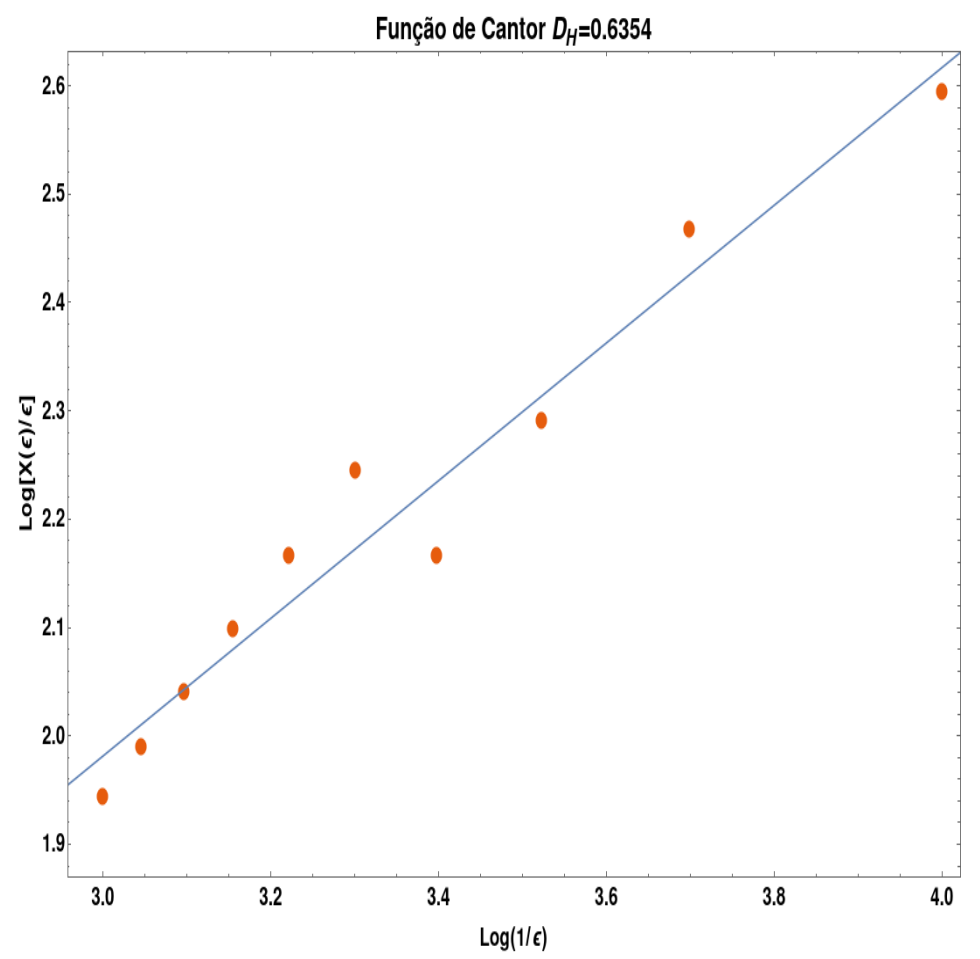

Figura 3.8: Ajuste para obter a dimensão de Hausdorff da função de Cantor. Obtivemos o valor 0,6354 .

Nas figuras anteriores foram utilizados valores de $\epsilon$ na escala de $10^{-4}$. Comparando com o valor razoável de 0,6309 [21], temos um resultado aceitavel dentro de uma incerteza na escala de $10^{-3}$. Nas figuras seguintes (3.9 e 3.10) são dados dois exemplos de ajustes feitos para o DM6 com duas temperaturas diferentes. 


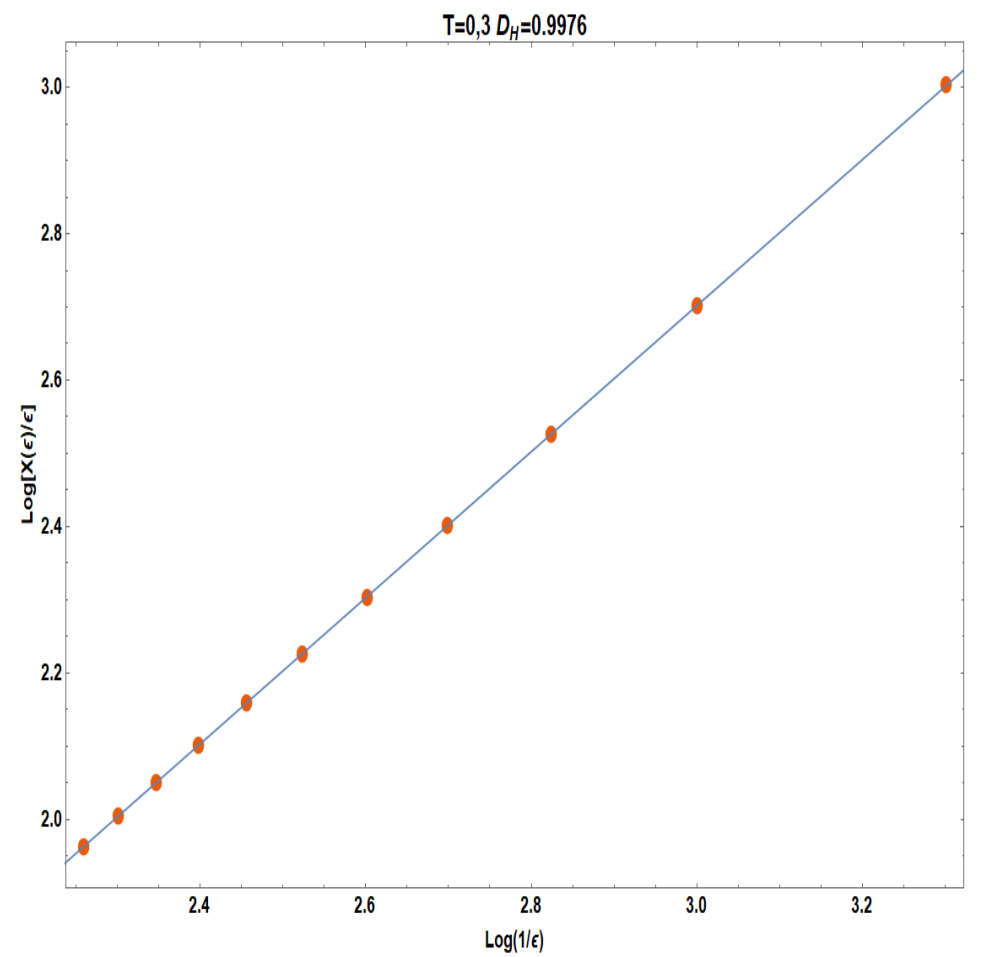

Figura 3.9: Ajuste da dimensão fractal para a temperatura $k_{B} T / J=0,3$. Obtivemos $D_{H} \approx$ 0,9976 .

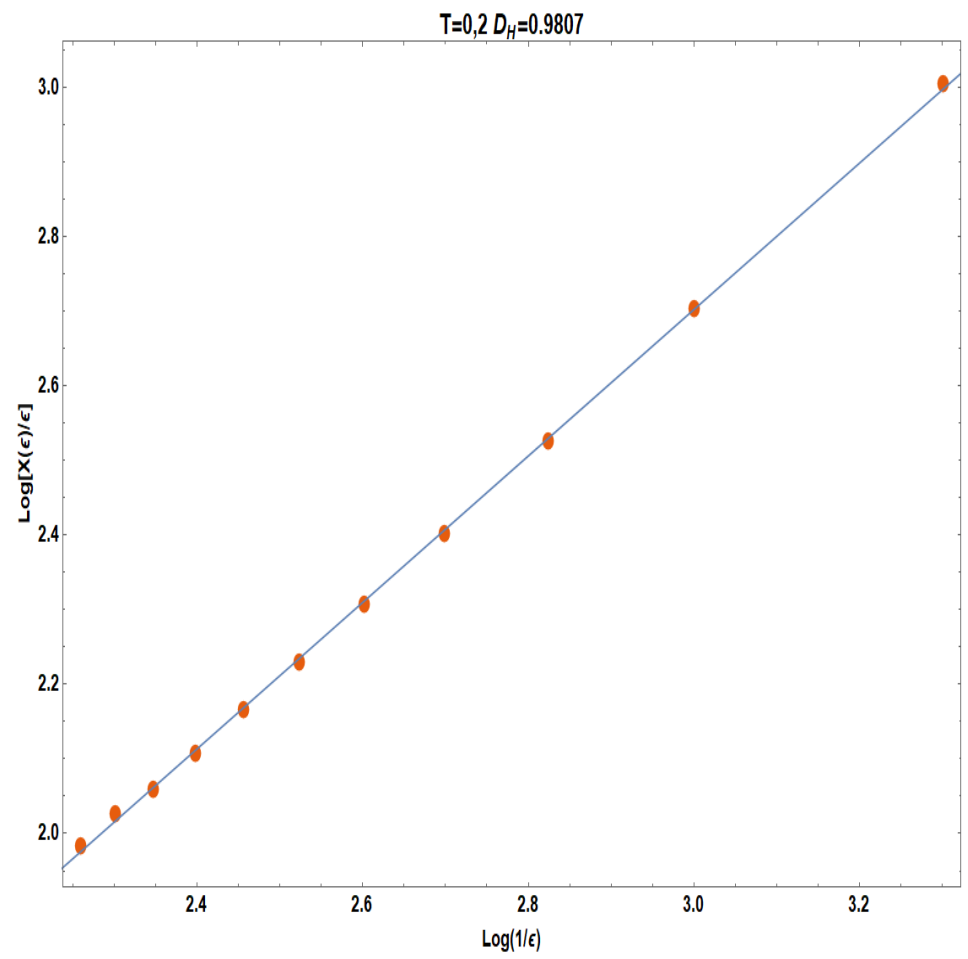

Figura 3.10: Ajuste da dimensão fractal para a temperatura $k_{B} T / J=0,2$. Obtivemos $D_{H} \approx$ 0,9807 . 
Os ajustes de melhor precisão refletem o fato de que a escada do modelo não é um fractal de fato, ou seja, não existem "degraus"arbitrariamente pequenos, ao contrário do que ocorre na função de Cantor. Para cada valor de $k_{B} T / J$ existe um valor mínimo para o tamanho do degrau; escolhendo $\epsilon$ da ordem desse tamanho um ajuste bem preciso foi alcançado [21].

Obtendo valores da dimensão para diferentes temperaturas, na mesma região de $p$, o resultado da figura 3.11 foi obtido. Nela se pode observar a tendência da dimensão aumentar com a temperarura, mas sempre menor que a unidade.

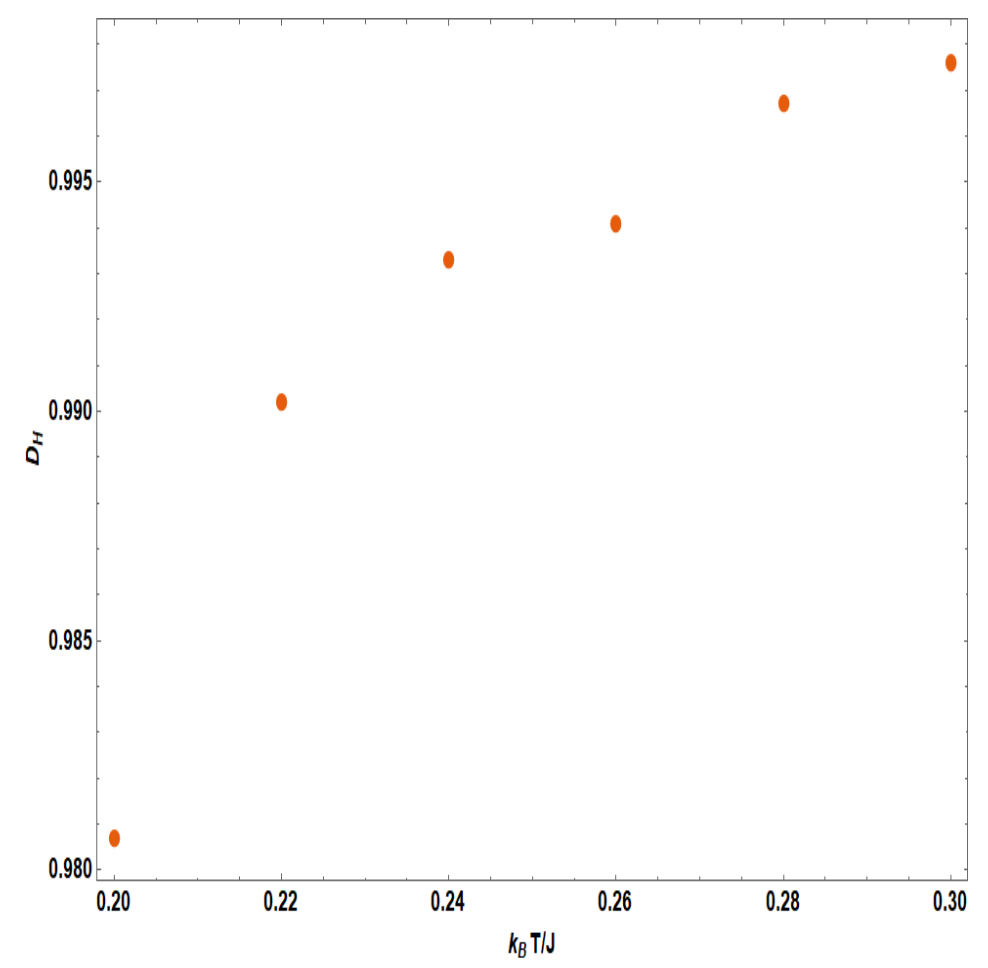

Figura 3.11: Valor da dimensão de Hausdorff $\left(D_{H}\right)$ contra a temperatura em unidades normalizadas $\left(k_{B} T / J\right)$.

Com esses resultados chegamos a algumas conclusões sobre o comportamento do sistema. Sabemos que em $k_{B} T / J=1 / 3$ o sistema começa a se tornar desordenado para certos valores de $T$. Variando a temperatura próximo desse ponto de transição e analisando as escadas para cada variação, percebemos que os degraus das frequências se tornam cada vez menores com o aumento da temperatura até se degenerarem. Com os degraus diminuindo, a dimensão do sistema se aproxíma de 1, e a figura 3.11 parece sugerir que esse processo se torna exponecialmente mais forte conforme a temperatura se aproxima da linha de transição para a fase desordenada. 


\subsection{Modelo DM6 - solução de campo médio}

A seguir descrevemos uma solução convencional de campo médio do modelo DM6, definido numa rede cúbica simples, com o eixo de quiralidade ao longo da direção z. Aproximações desse tipo foram utilizadas de forma pioneira para analisar o diagrama de fases do modelo ANNNI e posteriormente para obter a estrutura de fases dos modelos quirais do relógio [4].

Utilizando uma notação mais explícita para designar os sítios de uma rede cúbica simples, vamos escrever

$$
\mathcal{H}_{D M 6}=\mathcal{H}_{1}+\mathcal{H}_{2}
$$

com

$$
\mathcal{H}_{1}=-J_{0} \sum_{z=1}^{N} \sum_{\left(x, y ; x^{\prime} y^{\prime}\right)}\left[S_{x, y, z}^{x} S_{x^{\prime}, y^{\prime}, z}^{x}+S_{x, y, z}^{y} S_{x^{\prime}, y^{\prime}, z}^{y}+S_{x, y, z}^{z} S_{x^{\prime}, y^{\prime}, z}^{z}\right],
$$

em que $\left(x, y ; x^{\prime} y^{\prime}\right)$ designa um par de vizinhos mais próximos num plano normal à direção $z, \mathrm{e}$

$$
\begin{gathered}
\mathcal{H}_{2}=-J \sum_{x, y} \sum_{z=1}^{N}\left[S_{x, y, z}^{x} S_{x, y, z+1}^{x}+S_{x, y, z}^{y} S_{x, y, z+1}^{y}+S_{x, y, z}^{z} S_{x, y, z+1}^{z}\right]- \\
-D \sum_{x, y} \sum_{z=1}^{N}\left[S_{x, y, z}^{x} S_{x, y, z+1}^{y}-S_{x, y, z}^{y} S_{x, y, z+1}^{x}\right],
\end{gathered}
$$

em que a primeira soma (sobre $x$ e $y$ ) percorre todos os $N^{2}$ sítios do plano $(x, y)$, e a segunda soma (sobre $z$ ) leva em conta as interações de primeiros vizinhos ao longo da direção $z$.

Nessa etapa utilizamos a "desigualdade de Bogoliubov",

$$
G(\mathcal{H}) \leq G_{0}\left(\mathcal{H}_{0}\right)+\left\langle\mathcal{H}-\mathcal{H}_{0}\right\rangle_{0}=\Phi
$$

em que $G(\mathcal{H})$ é a energia livre associada ao hamiltoniano $\mathcal{H}, G_{0}\left(\mathcal{H}_{0}\right)$ é a energia livre associada a um hamiltoniano de tentativa $\mathcal{H}_{0}, \mathrm{e}\langle\ldots\rangle_{0}$ é um valor esperado obtido num ensemble canônico definido pelo hamiltoniano de tentativa. Com o objetivo de preservar a simetria das soluções, foi escolhido um hamiltoniano de tentativa por camadas, dado por

$$
\mathcal{H}_{0}=-\sum_{x, y} \sum_{z} \vec{\eta}_{z} \cdot \vec{S}_{x, y, z}=-\sum_{x, y} \sum_{z}\left[\eta_{z}^{x} S_{x, y, z}^{x}+\eta_{z}^{y} S_{x, y, z}^{y}+\eta_{z}^{z} S_{x, y, z}^{z}\right]
$$

em que $\vec{\eta}_{z}=\left(\eta_{z}^{x}, \eta_{z}^{y}\right)$ é um campo de tentativa bidimensional. Obtivemos a solução de campo médio pela minimização da energia livre de tentativa $\Phi$ em relação a $\left\{\vec{\eta}_{z}\right\}$. Não é difícil mostrar que

$$
G_{0}=-\frac{N^{2}}{\beta} \sum_{z} \ln \left[2 \cosh \left(\beta \eta_{z}^{x}\right)+2 \cosh \left(\beta \eta_{z}^{y}\right)+2 \cosh \left(\beta \eta_{z}^{z}\right)\right]
$$


e que

$$
\begin{gathered}
\frac{1}{N^{2}}\left\langle\mathcal{H}-\mathcal{H}_{0}\right\rangle_{0}=-2 J_{0} \sum_{z}\left[\left(m_{z}^{x}\right)^{2}+\left(m_{z}^{y}\right)^{2}+\left(m_{z}^{z}\right)^{2}\right]- \\
-J \sum_{z}\left[m_{z}^{x} m_{z+1}^{x}+m_{z}^{y} m_{z+1}^{y}+m_{z}^{z} m_{z+1}^{z}\right]- \\
-D \sum_{z}\left[m_{z}^{x} m_{z+1}^{y}-m_{z}^{y} m_{z+1}^{x}\right]+\sum_{z}\left[\eta_{z}^{x} m_{z}^{x}+\eta_{z}^{y} m_{z}^{y}+\eta_{z}^{z} m_{z}^{z}\right],
\end{gathered}
$$

em que usamos as definições

$$
\begin{aligned}
m_{z}^{x} & =\frac{\sinh \left(\beta \eta_{z}^{x}\right)}{\cosh \left(\beta \eta_{z}^{x}\right)+\cosh \left(\beta \eta_{z}^{y}\right)+\cosh \left(\beta \eta_{z}^{z}\right)} . \\
m_{z}^{y} & =\frac{\sinh \left(\beta \eta_{z}^{y}\right)}{\cosh \left(\beta \eta_{z}^{x}\right)+\cosh \left(\beta \eta_{z}^{y}\right)+\cosh \left(\beta \eta_{z}^{z}\right)} .
\end{aligned}
$$

$\mathrm{e}$

$$
m_{z}^{z}=\frac{\sinh \left(\beta \eta_{z}^{z}\right)}{\cosh \left(\beta \eta_{z}^{x}\right)+\cosh \left(\beta \eta_{z}^{y}\right)+\cosh \left(\beta \eta_{z}^{z}\right)} .
$$

Com esses resultados, utilizamos as definições dadas pelas equações (3.61) a (3.63) para escrever $\Phi$ na forma de uma série de potências nas magnetizações, fazendo contato com uma expansão do tipo Landau-Ginzburg. Mantendo apenas termos até segunda ordem nas magnetizações, é fácil mostrar que

$$
\begin{gathered}
\frac{1}{N^{2}} \Phi=-\frac{1}{\beta} N \ln 6+\sum_{z}\left(-2 J_{0}+\frac{2}{\beta}\right)\left[\left(m_{z}^{x}\right)^{2}+\left(m_{z}^{y}\right)^{2}+\left(m_{z}^{z}\right)^{2}\right]- \\
-J \sum_{z}\left[m_{z}^{x} m_{z+1}^{x}+m_{z}^{y} m_{z+1}^{y}+m_{z}^{z} m_{z+1}^{z}\right]-D \sum_{z}\left[m_{z}^{x} m_{z+1}^{y}-m_{z}^{y} m_{z+1}^{x}\right]+\cdots
\end{gathered}
$$

Para diagonalizar essa forma quadrática, vamos utilizar uma representação de Fourier. Escrevemos então

$m_{z}^{x}=\frac{1}{\sqrt{N}} \sum_{q} m_{q}^{x} \exp (i q z) ; \quad m_{z}^{y}=\frac{1}{\sqrt{N}} \sum_{q} m_{q}^{y} \exp (i q z) ; \quad m_{z}^{z}=\frac{1}{\sqrt{N}} \sum_{q} m_{q}^{z} \exp (i q z)$,

com as somas restritas à zona de Brillouin. Também é interessante escrever $m_{q}^{x}, m_{q}^{y}$ e $m_{q}^{z}$ em termos de suas partes reais e imaginárias,

$$
m_{q}^{x}=\frac{1}{\sqrt{2}}\left(R_{q}^{x}+i I_{q}^{x}\right), \quad m_{q}^{y}=\frac{1}{\sqrt{2}}\left(R_{q}^{y}+i I_{q}^{y}\right), \quad m_{q}^{z}=\frac{1}{\sqrt{2}}\left(R_{q}^{z}+i I_{q}^{z}\right) .
$$

Levando em conta que as magnetizações são reais, e que a zona de Brillouin é simétrica, também temos

$$
R_{q}=R_{-q}, \quad I_{q}=-I_{-q}
$$


para as componentes $x, y$ e $z$. Então não é difícil mostrar que

$$
\begin{gathered}
\frac{1}{N^{2}} \Phi=-\frac{1}{\beta} N \ln 6+ \\
+\frac{1}{2} \sum_{q}\left(\frac{2}{\beta}-2 J_{0}-J \cos q\right)\left[\left(R_{q}^{x}\right)^{2}+\left(I_{q}^{x}\right)^{2}+\left(R_{q}^{y}\right)^{2}+\left(I_{q}^{y}\right)^{2}+\left(R_{q}^{z}\right)^{2}+\left(I_{q}^{z}\right)^{2}\right]- \\
-D \sum_{q}(\operatorname{sen} q)\left[I_{q}^{x} R_{q}^{y}-I_{q}^{y} R_{q}^{x}\right]+\cdots
\end{gathered}
$$

Portanto, até termos de segunda ordem, a energia livre de tentativa fica expressa em termos de modos reais e independentes no espaço de Fourier, mas acoplados nos planos $x-y$.

Definimos agora as grandezas

$$
A=\frac{1}{\beta}-J_{0}-\frac{1}{2} J \cos q ; \quad B=D \operatorname{sen} q
$$

e utilizamos a notação simplificada

$$
x_{1}=R_{q}^{x} ; \quad x_{2}=I_{q}^{x} ; \quad y_{1}=R_{q}^{y} ; \quad y_{2}=I_{q}^{y} \quad z_{1}=R_{q}^{z} ; \quad z_{2}=I_{q}^{z}
$$

Portanto, temos a forma quadrática que deve ser analisada,

$$
Q=A\left(x_{1}^{2}+x_{2}^{2}+y_{1}^{2}+y_{2}^{2}+z_{1}^{2}+z_{2}^{2}\right)+B\left(y_{2} x_{1}-x_{2} y_{1}\right)=(\vec{v})^{t} \mathbf{M}(\vec{v})
$$

em que $\vec{v}$ é um vetor coluna, $\widetilde{\mathbf{v}}=\left(x_{1}, x_{2}, y_{1}, y_{2}, z_{1}, z_{2}\right)$, e a matriz $\mathbf{M}$ é dada por

$$
\mathbf{M}=\left(\begin{array}{cccccc}
A & 0 & 0 & B / 2 & 0 & 0 \\
0 & A & -B / 2 & 0 & 0 & 0 \\
0 & -B / 2 & A & 0 & 0 & 0 \\
B / 2 & 0 & 0 & A & 0 & 0 \\
0 & 0 & 0 & 0 & A & 0 \\
0 & 0 & 0 & 0 & 0 & A
\end{array}\right)
$$

Essa matriz $\mathrm{M}$ tem dois autovalores triviais degenerados,

$$
\lambda_{1}=\lambda_{2}=A=\frac{3}{4 \beta}-J_{0}-\frac{1}{2} J \cos q .
$$

Para encontrar os outro quatro autovalores temos que diagonalizar a matriz supersimétrica

$$
\mathbf{M}_{r}=\left(\begin{array}{cccc}
A & 0 & 0 & B / 2 \\
0 & A & -B / 2 & 0 \\
0 & -B / 2 & A & 0 \\
B / 2 & 0 & 0 & A
\end{array}\right)
$$


que é muito semelhante à matriz análoga associada ao cálculo de campo médio do modelo DM4. Os autovalores degenerados dessa matriz são dados por

$$
\lambda_{3,4}=A \pm \frac{1}{2} B=\frac{3}{4 \beta}-J_{0}-\frac{1}{2} J \cos q \pm \frac{1}{2} D \operatorname{sen} q
$$

Considerando a forma desses autovalores, a fronteira crítica da fase desordenada será dada por

$$
\frac{1}{\beta_{c}}=k_{B} T_{c}=\max _{q}\left[\frac{2}{3} J_{0}+\frac{1}{3} J \cos q \pm \frac{1}{3} D \operatorname{sen} q\right] .
$$

Para $D=0$, recuperamos a temperatura crítica do ferromagneto usual,

$$
\frac{1}{\beta_{c}}=k_{B} T_{c}=\max _{q}\left(\frac{2}{3} J_{0}+\frac{1}{3} J \cos q\right)=\frac{2}{3} J_{0}+\frac{1}{3} J,
$$

pois $q_{c}=0$ é o primeiro valor do número de onda associado a um autovalor nulo da forma quadrática. Para $D \neq 0$, a maximação em relação ao número de onda fornece dois resultados

$$
\tan q= \pm \frac{D}{J}
$$

que correspondem a ondas propagantes nos dois sentidos do eixo $z$, com uma temperatura crítica dada por

$$
\frac{1}{\beta_{c}}=k_{B} T_{c}=\frac{2}{3} J_{0}+\frac{1}{3}\left(J^{2}+D^{2}\right)^{1 / 2}
$$

que deve ser comparada com a expressão análoga no limite de coordenação infinita da árvore de Cayley.

\subsection{Versão esférica do modelo de Dzyaloshinskii-Moriya}

Desde que foi proposto, por Berlin e Kac na década de 1950, o modelo esférico do ferromagnetismo, que admite solução exata em qualquer dimensão, até mesmo na presença de campo externo, tem sido muito utilizado como uma espécie de laboratório ou campo de prova do comportamento de sistemas mais realistas. Em geral, o modelo esférico prevê um comportamento crítico distinto das aproximações de campo médo, mas distinto também dos resultados experimentais.

A função canônica de partição do modelo de Ising pode ser escrita na forma

$$
Z_{I}=\sum_{\left\{\sigma_{i}\right\}} \exp \left[\beta J \sum_{(i, j)} \sigma_{i} \sigma_{j}\right]
$$


em que estamos usando a notação usual ( $\beta$ é o inverso da temperatura e $J$ é o parâmetro de troca) e as variáves de spin, situadas nos sítios de uma rede cristalina podem assumir apenas dois valores (ou seja, $\sigma_{i}= \pm 1$, para $i=1,2, \ldots, N$ ). A primeria soma é sobre todas as configurações desse sistema (todos os valores das variáves de spin). A segunda soma é sobre vizinhos mais próximos na rede. Esse é o problema famoso que Onsager resolveu exatamante na rede quadrada, mostrando que no limite termodinâmico pode ocorrer uma divergência logarítmica do calor específico com a temperatura. No momento, continuamos na expetativa de soluções exatas na presença de um campo externo ou em redes tridimensionais (em uma dimensão, Ernst Ising, em 1924, mostrou que esse modelo não tem nenhuma anomalia termodinâmica).

A ideia de Berlin e Kac consistiu em deformar o modelo de Ising, considerando um sistema matematicamente mais acessível, em que as variáveis de spin, do tipo $\sigma_{i}= \pm 1$, são substituídas por variáveis contínuas, $-\infty \leq \sigma_{i} \leq+\infty$, percorrendo todo o eixo real. Para evitar uma divergência da função de partição, Berlin e Kac introduziram uma "condição esférica",

$$
\sum_{i=1}^{N} \sigma_{i}^{2}=N
$$

que também e obedecida pelo modelo de Ising, e escreveram a função canônica de partição do modelo esférico,

$$
Z_{E}=\prod_{i=1}^{N}\left[\int_{-\infty}^{+\infty} d \sigma_{i}\right] \exp \left[\beta J \sum_{(i, j)} \sigma_{i} \sigma_{j}\right] \delta\left[\sum_{i=1}^{N} \sigma_{i}^{2}-N\right]
$$

em que $\delta(. .$.$) é uma função de Dirac. Usando uma representação integral da função \delta$, é simples realizar as integrações sobre as variáveis de spin, e reduzir o problema ao cálculo de uma única integral, que pode ser obtida pela aplicação do método do ponto de sela.

Há outra abordagem desse problema, um pouco mais simples, que consiste em definir um "modelo esférico médio", associado a uma espécie de função grande canônica de partição,

$$
\Xi_{E M}=\prod_{i=1}^{N}\left[\int_{-\infty}^{+\infty} d \sigma_{i}\right] \exp \left[\beta J \sum_{(i, j)} \sigma_{i} \sigma_{j}\right] \exp \left[-\mu \sum_{i=1}^{N} \sigma_{i}^{2}\right]=\Xi[\beta, \mu]
$$

em que $\mu$ é um potencial químico, dado pela condição

$$
\langle N\rangle=-\frac{\partial}{\partial \mu} \ln \Xi
$$

Nessa seção, vamos apresentar um esboço de cálculo, de natureza ainda preliminar, para um modelo esférico médio de três componentes distintas. O nosso trabalho foi em parte motivado 
por artigo relativamente recente de Aqua e Fisher[22], que consideraram um modelo de gás de rede com diversos componentes, cada um deles sujeito a um vínculo esférico. Modelos esféricos ferromagnéticos de vários componentes já tinham sido analisados na literatura anterior, resultando apenas numa fatorização da função grande canônica de partição. Uma fatorizaçao desse tipo também foi notada por Aqua e Fisher. No caso em que estamos interessados, no entanto, as interações monoaxiais do tipo Dzyaloshinskii-Moriya produzem um acoplamento entre dois tipos de variáveis de spin, tornando o problema bem mais interessante [23] .

Vamos então considerar uma versão bem simples do modelo DM6, consistindo num hamiltoniano ferromagnético de Heisenberg, com interaçoes restritas aos sítios vizinhos mais próximos numa rede cúbica simples, com a adição de uma interação do tipo Dzyaloshinskii-Moriya ao longo do eixo $z$. O hamiltoniano desse sistena é dado por

$$
\mathcal{H}=-J \sum_{\left(\vec{r}, \vec{r}^{\prime}\right)} \vec{S}_{\vec{r}} \cdot \vec{S}_{\vec{r}^{\prime}}-D \sum_{\vec{r}}\left(\vec{S}_{\vec{r}} \times \vec{S}_{\vec{r}+\hat{z}}\right) \cdot \hat{z}
$$

em que $J>0, \vec{r}$ designa os sítios de uma rede cúbica simples de $N$ sítios, a primeira soma é sobre sítios vizinhos mais próximos, e a segunda é sobre vizinhos na direção $z$, em que $\widehat{z}$ designa um vetor unitário da rede ao longo da direção axial. Estamos considerando três compontes de spin, $\vec{S}_{\vec{r}}=\left(S_{\vec{r}}^{x}, S_{\vec{r}}^{y}, S_{\vec{r}}^{z}\right)$, em que cada componente pode variar no eixo real, mas sujeita ao vínculo esférico médio.

A grande função de partição desse sistema é dada pela integral

$$
\left.\Xi=\prod_{\vec{r}} \int_{-\infty}^{+\infty} d S_{\vec{r}}^{x} \int_{-\infty}^{+\infty} d S_{\vec{r}}^{y} \int_{-\infty}^{+\infty} d S_{\vec{r}}^{z} \exp \left[-\beta \mathcal{H}-\mu_{1} \sum_{\vec{r}}\left(S_{\vec{r}}^{x}\right)^{2}-\mu_{2} \sum_{\vec{r}}\left(S_{\vec{r}}^{y}\right)^{2}-\mu_{3} \sum_{\vec{r}}\left(S_{\vec{r}}^{z}\right)^{2}\right]\right],
$$

com os vínculos esféricos médios,

$$
\left\langle\left(S_{\vec{r}}^{\alpha}\right)^{2}\right\rangle=-\frac{\partial}{\partial \mu_{\alpha}} \ln \Xi=N,
$$

em que $\alpha=x, y, z$. Adotando condições periódicas de contorno, podemos introduzir a representação de Fourier,

$$
\vec{S}_{\vec{r}}=\frac{1}{\sqrt{N}} \sum_{\vec{q}} \vec{S}_{\vec{q}} \exp [i \vec{q} \cdot \vec{r}]
$$

em que a soma deve ser feita sobre uma zona de Brillouin simétrica e elementar. Levando em consideração que as variáveis de spin são reais, também temos

$$
\vec{S}_{\vec{q}}=\left(\vec{S}_{-\vec{q}}\right)^{*}
$$


Então, não é difícil mostrar que

$$
\begin{gathered}
\overline{\mathcal{H}}=\beta J \sum_{\vec{q}}\left(\cos q_{x}+\cos q_{y}+\cos q_{z}\right)\left(S_{\vec{q}}^{x} S_{-\vec{q}}^{x}+S_{\vec{q}}^{y} S_{-\vec{q}}^{y}+S_{\vec{q}}^{z} S_{-\vec{q}}^{z}\right)+ \\
+2 \beta D i \sum_{\vec{q}}\left(\operatorname{sinq}_{z}\right) S_{\vec{q}}^{x} S_{-\vec{q}}^{y}-\mu_{1} \sum_{\vec{q}}\left(S_{\vec{q}}^{x} S_{-\vec{q}}^{x}\right)-\mu_{2} \sum_{\vec{q}}\left(S_{\vec{q}}^{y} S_{-\vec{q}}^{y}\right)-\mu_{3} \sum_{\vec{q}}\left(S_{\vec{q}}^{z} S_{-\vec{q}}^{z}\right) .
\end{gathered}
$$

Vamos agora introduzir as variáveis reais $\left(R_{q}^{\alpha}, I_{q}^{\alpha}\right), \operatorname{com} \alpha=x, y, z$, tal que

$$
S_{\vec{q}}^{\alpha}=\frac{1}{\sqrt{2}}\left(R_{q}^{\alpha}+i I_{q}^{\alpha}\right)
$$

em que simplificamos um pouco a notação vetorial (e vamos tratar separadamente o caso $q=0$ ). É fácil verificar que

$$
R_{\vec{q}}^{\alpha}=R_{-\vec{q}}^{\alpha}, \quad I_{q}^{\alpha}=-I_{-q}^{\alpha} .
$$

Portanto, com mais algumas manipulações algébricas, e descartando o termo dependendente de $q=0$, obtemos uma forma quadrática,

$$
\begin{aligned}
\overline{\mathcal{H}}= & \beta J \sum_{\vec{q}>0}\left(\cos q_{x}+\cos _{y}+\cos q_{z}\right)\left[\left(R_{\vec{q}}^{x}\right)^{2}+\left(I_{\vec{q}}^{x}\right)^{2}+\left(R_{\vec{q}}^{y}\right)^{2}+\left(I_{\vec{q}}^{y}\right)^{2}+\left(R_{\vec{q}}^{z}\right)^{2}+\left(I_{\vec{q}}^{z}\right)^{2}\right]+ \\
& +2 \beta D \sum_{\vec{q}>0}\left(\sin q_{z}\right)\left[R_{\vec{q}}^{x} I_{\vec{q}}^{y}-R_{\vec{q}}^{y} I_{\vec{q}}^{x}\right]- \\
& -\mu_{1} \sum_{\vec{q} \geq 0}\left[\left(R_{\vec{q}}^{x}\right)^{2}+\left(I_{\vec{q}}^{x}\right)^{2}\right]-\mu_{2} \sum_{\vec{q} \geq 0}\left[\left(R_{\vec{q}}^{y}\right)^{2}+\left(I_{\vec{q}}^{y}\right)^{2}\right]-\mu_{3} \sum_{\vec{q} \geq 0}\left[\left(R_{\vec{q}}^{z}\right)^{2}+\left(I_{\vec{q}}^{z}\right)^{2}\right]
\end{aligned}
$$

em que ainda mantivemos três potenciais químicos distintos.

Para dar continuidade a essa análise verificamos que é suficiente considerar duas formas quadráticas. Uma delas, envolvendo apenas componentes segundo z, é trivial, pois já está diagonalizada,

$$
Q_{1}=\left[\beta J\left(\cos q_{x}+\cos q_{y}+\cos q_{z}\right)-\mu_{3}\right]\left[\left(R_{\vec{q}}^{z}\right)^{2}+\left(I_{\vec{q}}^{z}\right)^{2}\right]
$$

Essa forma quadrática indica que devemos ter

$$
\mu_{3}>\beta J \max _{\vec{q}}\left(\cos q_{x}+\cos q_{y}+\cos q_{z}\right)
$$

que é a condição usual para um ordenamento ferromagnético simples.

Simplificando a notação, a outra forma quadrática é dada por

$$
Q_{2}=A\left(x_{1}^{2}+y_{1}^{2}\right)+C\left(x_{2}^{2}+y_{2}^{2}\right)+B\left(x_{1} y_{2}-y_{1} x_{2}\right)
$$


com

$$
\begin{gathered}
A=\beta J\left(\cos q_{x}+\cos q_{y}+\cos q_{z}\right)-\mu_{1}, \\
B=2 \beta D \sin q_{z},
\end{gathered}
$$

e

$$
C=\beta J\left(\cos q_{x}+\cos q_{y}+\cos q_{z}\right)-\mu_{2},
$$

em que $x_{1}=R_{q}^{x}, y_{1}=I_{q}^{x}, x_{2}=R_{q}^{y}, y_{2}=R_{q}^{y}$. Para analisar essa fora quadrática, torna-se conveniente escrever a matriz $4 \times 4$,

$$
M=\left(\begin{array}{cccc}
A & 0 & 0 & \frac{1}{2} B \\
0 & A & -\frac{1}{2} B & 0 \\
0 & -\frac{1}{2} B & C & 0 \\
\frac{1}{2} B & 0 & 0 & C
\end{array}\right) .
$$

Note que essa matriz é simétrica, com auotovalores reais, e que apenas não é totalmente trivial devido ao termo $B \neq 0$, associado à quiralidade. Agora é fácil escrever dois autovalores degenerados,

$$
\Lambda=\beta J\left(\cos q_{x}+\cos q_{y}+\cos q_{z}\right)-\frac{1}{2}\left(\mu_{1}+\mu_{3}\right) \pm \frac{1}{2}\left[\left(\mu_{1}-\mu_{2}\right)^{2}+4 \beta^{2} D^{2} \sin ^{2} q_{z}\right]^{1 / 2}
$$

Há uma série de consequências dessas expressões. Por exemplo, supondo que $\mu_{1}=\mu_{2}=\mu$, devemos ter

$$
\mu>\beta J \max _{\vec{q}}\left\{\left(\cos q_{x}+\cos q_{y}+\cos q_{z}\right) \pm d \sin q_{z}\right\},
$$

em que estamos tomando $d=D / J>0$. Essa condição se verifica para $q_{x}=q_{y}=0$, ou seja, ordenamento planar, mas

$$
\tan q_{z}= \pm \frac{D}{J}
$$

que é uma situação típica de ondas espaciais de magnetização ao longo das duas direções do eixo $z$, como já tínhamos encontrado na solução de campo médio.

Por enquanto, conseguimos obter esses resultados, que já funcionam para indicar a existência de estruturas moduladas, em conformidade com as previsões da aproximação de campo médio. Pretendemos dar andamento a esse trabalho, realizando uma análise bem detalhada, necessariamente numérica [24], para obter as linhas críticas num diagrama de fases em termos da temperatura $T$ e do parâmetro de quiralidade $d$.

Ao invés de prosseguir na análise mais envolvida do modelo esférico, poderíamos ter considerado uma aproximação bem mais simples, uma "versão gaussiana" desse mesmo modelo, 
que infelizmente só existe acima da temperatura crítica, como foi originalmente apontado por Berlin e Kac (vamos então dar um passo atrás). Essa versão gaussiana pode ser obtida a partir dos nossos cálculos simplesmente fazendo

$$
\mu_{1}=\mu_{2}=\mu_{3}=b>0
$$

em que $b>0$ é uma constante positiva (para simplificar, vamos tomar $b=1$ ). Então, nessa aproximação gaussiana temos:

$$
\text { (i) } \frac{1}{T}\left(\cos q_{x}+\cos _{y}+\cos q_{z}\right)-1<0
$$

de onde vem o "ramo ferromagnético"da temperatura crítica,

$$
T_{c 1}=\max _{\vec{q}}\left\{\left(\cos q_{x}+\cos q_{y}+\cos q_{z}\right)\right\}=3
$$

e

$$
\text { (ii) } \frac{1}{T}\left(\cos q_{x}+\cos q_{y}+\cos q_{z}\right)-1 \pm \frac{d}{T} \sin q_{z}
$$

de onde vem o "ramo modulado" da temperatura crítica,

$$
T_{c 2}=\max _{\vec{q}}\left[\cos q_{x}+\cos q_{y}+\cos q_{z} \pm d \sin q\right]
$$

ou seja

$$
T_{c 2}=2+\left(1+d^{2}\right)^{1 / 2},
$$

que se realiza para $q_{x}=q_{y}=0$, mas $\tan q_{z}= \pm D / J$, que corresponde aos resultados anteriores. Como $T_{c 2}>T_{c 1}$, exceto para $d=0$, de acordo com as indicações do nosso cálculo de campo médio, a transição sempre se dá para uma fase modulada, com duas ondas espaciais propagantes ao longo do eixo $z$. 


\section{Capítulo 4}

\section{Conclusões}

Todos os resultados explorados nos capítulos anteriores indicam a existência de fases moduladas para um sistema do tipo Heisenberg com interações do tipo DM. Essas fases produzem ajustes com estruturas de natureza fractal, com dimensões menores do que um, que chamamos de "escadas do diabo". É importante notar que neste trabalho só estudamos as fases chamadas "comensuráveis", ou seja, aquelas em que a frequência da modulação tem uma razão racional com o parâmetro de estrutura da rede.

As estruturas incomensuráveis provavelmente existem nas regiões de transição entre as fases comensuráveis [5]. Na figura 4.1 abaixo exibimos uma investigação mais detalhada da região modulada do diagrama de fases do DM6. As regiões mais escuras são fases comensuráveis com um certo valor fixo de frequência; as regiões claras são as transições entre essas fases; nessas regiões possivelmente existem fases incomensuráveis. 


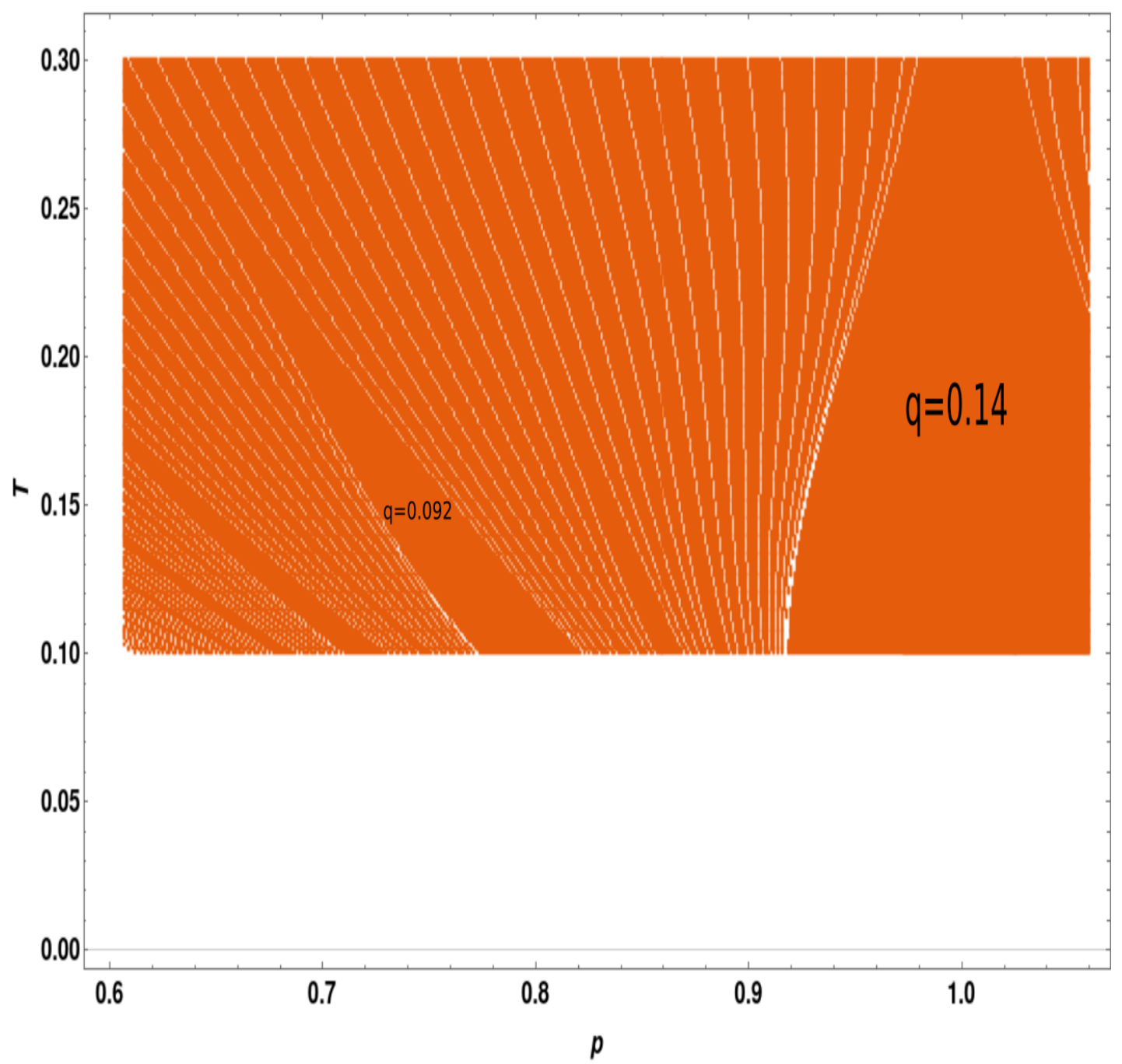

Figura 4.1: Diagrama mais detalhado de uma parte da região modulada do modelo DM6. As faixas verticais mais escuras representam fases comensuráveis e as regiões claras são suas linhas de transição.

É notável também a presença de uma grande fase comensurável ao redor de $p=1$. Isso se relaciona com o estudo feito em uma dimensão que aponta a existência de uma entropia residual para este valor do parâmetro $\mathrm{p}$.

Também é importante comentar sobre as equações de campo médio. Elas nos fornecem detalhes mais precisos sobre as fronteiras de transição. Além disso, podem determinar a fase dominante quando há mais de uma possibilidade de atrator, por meio da energia livre, uma grandeza que não podemos obter da árvore de Cayley. Porém, como foi visto, as equações na árvore de Cayley são muito mais simples e nos permitem ganhar muito tempo, principalmente quanto a esforços computacionais. Pelo menos sob o ponto de vista qualitativo, os cálculos na 
árvore de Cayley são muito instrutivos.

Por fim, no futuro desejamos desenvolver ainda mais as equações do modelo esférico para o DM6. Pretendemos também analisar uma "versão quântica"do modelo esferíco e fazer conexões com cálculos relativamente recentes para modelos quânticos com interações quirais. 


\section{Referências Bibliográficas}

[1] J. Yeomans, M. E. Fisher, J. Phys. C, 14, L835, 1981.

[2] J. Yeomans, Solid State Physics, 41, 151, 1988.

[3] W. Selke, Phys. Repts., 170, 213, 1988; W. Selke, em C. Domb e J. L. Lebowitz, Phase Transitions and Critical Phenomena, 15, Academic Press, Londres, 1992.

[4] C. S. O. Yokoi, M. D. Coutinho-Filho, S. R. Salinas, Phys. Rev. B, 24, 4047, 1981.

[5] P. Bak, Rep. Prog. Phys., 45, 587, 1982.

[6] I. Dzyaloshinsky, J. Phys. Chem. Solids., 4, 241, 1958.

[7] T. Moriya, Phys. Review, 120, 91, 1960.

[8] Yu. A. Izyumov, Sov. Phys. Usp., 27, 845, 1984.

[9] Z. Saghafi, J.Jahangiri, S. Mahdavifar, H. Hadipour, S. Farjami Shayesteh, Journal of Magnetism and Magnetic Materials, 398, 183, 2016.

[10] M. Schmitt, P. Moras, G. Bihlmayer, R. Cotsakis, M. Vogt, J. Kemmer, A. Belabbes, M. P. Sheverdyaeva, A. K. Kundu, C. Carbone, S. Blügel, M. Bode ,Nature Communications, 10, 2610, 2019.

[11] Pylypovskyi, V. Oleksandr ; Kravchuk, Volodymyr P.; Volkov, Oleksii M.; Fabbender, Jürgen; Sheka, Denis D.; Makarov, Denys, eprint arXiv:2001.03408, 5 pages, 2020.

[12] M. Pleimling, B. Neubert, R. Siems, J. Phys. A, 31, 4875, 1998.

[13] J. D. Noh, H. Rieger, M. Enderle, K. Knorr Phys. Review E, 66, 026111, 2002.

[14] M. J. de Oliveira, S. R. Salinas, Revista Brasileira de Física, 15, 189, 1985. 
[15] C. J. Thompson, Journal of Statistical Physics, 27, 441, 1982.

[16] H. C. Ottinger, J. Phys. C, 16, L257 e L597, 1983.

[17] M. Siegert, H. U. Evans, Z. Phys. B, 60, 265, 1985.

[18] M. Shinozaki, S. Hoshino, Y. Masaki, J. Kishine, Y. Kato, J. Phys. Soc. Jpn, 85, 074710, 2016.

[19] E. S. Nascimento, J. P. de Lima, S. R. Salinas, Physica A, 409, 78, 2014.

[20] O. Dovgoshey, O. Martio, V. Ryazanov, M. Vuorinen, Expo. Math., 24, 1, 2006.

[21] Fiedler-Ferrara, Nelson, Cintra do Prado, CP, Caos:Uma introdução, Edgar Blucher, 1994.

[22] Jean-Noël Aqua, Michael E. Fisher, Phys. Rev. E, 24, 79, 2009.

[23] G. V. Bettoney, R. M. Mazo, Journal of Mathematical Physics, 11, 1147, 1970.

[24] Yokoi, Carlos S. O., dissertação de mestrado, IFUSP, 1978. 